\title{
A Comparative Analysis of Quantitative Metrics of Root Architecture
}

\author{
Harini Rangarajan and Jonathan P. Lynch $(\mathbb{D}$ \\ Department of Plant Science, The Pennsylvania State University, University Park, PA 16802, USA \\ Correspondence should be addressed to Jonathan P. Lynch; jpl4@psu.edu
}

Received 8 September 2020; Accepted 22 January 2021; Published 2 March 2021

Copyright (c) 2021 Harini Rangarajan and Jonathan P. Lynch. Exclusive Licensee Nanjing Agricultural University. Distributed under a Creative Commons Attribution License (CC BY 4.0).

\begin{abstract}
High throughput phenotyping is important to bridge the gap between genotype and phenotype. The methods used to describe the phenotype therefore should be robust to measurement errors, relatively stable over time, and most importantly, provide a reliable estimate of elementary phenotypic components. In this study, we use functional-structural modeling to evaluate quantitative phenotypic metrics used to describe root architecture to determine how they fit these criteria. Our results show that phenes such as root number, root diameter, and lateral root branching density are stable, reliable measures and are not affected by imaging method or plane. Metrics aggregating multiple phenes such as total length, total volume, convex hull volume, and bushiness index estimate different subsets of the constituent phenes; they however do not provide any information regarding the underlying phene states. Estimates of phene aggregates are not unique representations of underlying constituent phenes: multiple phenotypes having phenes in different states could have similar aggregate metrics. Root growth angle is an important phene which is susceptible to measurement errors when $2 \mathrm{D}$ projection methods are used. Metrics that aggregate phenes which are complex functions of root growth angle and other phenes are also subject to measurement errors when $2 \mathrm{D}$ projection methods are used. These results support the hypothesis that estimates of phenes are more useful than metrics aggregating multiple phenes for phenotyping root architecture. We propose that these concepts are broadly applicable in phenotyping and phenomics.
\end{abstract}

\section{Introduction}

Crop production needs to double by 2050 to provide for the increasing global population [1-4]. A major challenge is the identification of efficient crops that cope with climate change and reduce the need for fertilizer and water inputs to make agriculture environmentally sustainable. Root architecture influences water and nutrient uptake, so selecting and developing efficient crops based on their root system architecture (RSA) has been proposed as a strategy towards a "second green revolution" [5-8].

Development of powerful tools in genomic research has resulted in a deluge of genomic information. However, this genomic information cannot be fully exploited for crop improvement unless it is linked to the phenome [9-11]. In the context of roots, the root phenome is the set of phenes manifested by roots of a plant, where phenes are elementary units of the phenotype; phenes are related to phenotypes as genes are to genotypes $[10,12]$. Phenotyping is a bottleneck for breeding and genetic analysis because it is species-specific, labor intensive, and environmentally sensitive, unlike genotyping, which is uniform across organisms, highly automated, and increasingly inexpensive $[9,10,13,14]$. Phenotyping is especially challenging for roots because of their complexity, plasticity, and inaccessibility. Significant advances are being made in phenotyping methods and technology in an attempt to develop high-throughput platforms. In order to develop efficient strategies to explore the phenome, it is important to clarify what constitutes a phenotype, delineate the key components that comprise a phenotype, and determine the level of resolution at which phenotypic data must be collected. Although an essentially infinite number of measurements may be collected to describe each phenotype, a smaller number of more basic variables may explain most of the important phenotypic variation among genotypes. These basic variables or phenes are the elementary units of the root phenotype and cannot be decomposed to more phenes at the same scale of organization [10]. Based on this definition, number of axial roots, lateral root branching density (LRBD), root growth angle, root diameter, and root length of different root classes of the root system can be considered as phenes. 
Current methods for developing high-throughput phenotyping platforms and identification of relevant quantitative trait loci (QTL) associated with traits of interest are largely based on nonelementary phenotypic metrics. Nonphenes, referred to as phene aggregates in this paper, are aggregate components of the root phenotype and describe the distribution of roots, shape of roots, and/or size of the root system. Phene aggregates include several conventionally measured traits including total root length, total area, and total volume, as well as novel phenotypic metrics such as convex hull volume, convex hull area, ellipse major axis, ellipse minor axis, ellipse aspect ratio, volume distribution, solidity, bushiness index [9, 15-17], and metrics which measure the geometry and complexity of root systems such as fractal dimension (FD), fractal abundance (FA), and lacunarity [18-20]. Aggregate phenotypic metrics (referred to as aggregate metrics) are comprised of phenes; some of these can be measured as a simple aggregate of phenes (e.g., total length), some are represented as a function of other aggregates (e.g., bushiness, solidity, and volume distribution), some measure shapes resulting from interaction of the constituent phenes (e.g., Convex hull volume), and some metrics are complex metrics which measure emergent properties of root architecture and cannot be described as a simple aggregate, shape aggregate, or a function of other aggregates (e.g., Fractal Dimension).

Estimates of phene aggregates change over time and are phenotype specific. Some phene aggregates increase over time, some remain relatively static, and some decrease in value over time $[16,21]$. The magnitude of change in estimates of phene aggregates with time also varies greatly. This is because some of the phene aggregates are one-dimensional measurements while some measurements are a function of more than one dimension [22]. Many phene aggregates are estimates generated from the average values of the $2 \mathrm{D}$ projections in a rotational image series [17] and are thought to represent $3 \mathrm{D}$ root shape accurately. However, which traits can be measured accurately using estimates derived from $2 \mathrm{D}$ data and which require 3D representations is poorly understood. Depending on the phenotype, metrics derived from rotated $2 \mathrm{D}$ projections of the same $3 \mathrm{D}$ root system can vary significantly. This leads to a related question of how much should an aggregate phenotypic metric differ for two phenotypes to be considered distinctly different. Fractal analysis of corn roots has shown that the $F D$ of two genotypes can be the same but vary in FA [23]. Root systems with similar FD may vary functionally, and genotypes can be distinguished when fractal analysis involves FD, FA, and lacunarity [20]. Aggregate phene metrics estimate the aggregate of multiple phenes. For example, greater rooting depth is an important trait for capture of subsoil $\mathrm{N}$ in maize. Greater rooting depth results from a combination of deeper axial root growth angle [24-26], root elongation rate [27], expression of fewer crown roots $[28,29]$, reduced lateral branching density $[30,31]$, formation of root cortical aerenchyma (RCA) $[29,32]$, reduced cortical file number, and increased cortical size $[33,34]$. Each of these phenes is under distinct genetic control and has important interactions with each other. Selection for combination of specific phenes will therefore be much simpler and precise than would selection for root depth itself [7]. Phenes are under more simple genetic control and permit more precise control over the root system architecture (RSA) and so are more useful for selection for crop breeding $[7,10]$.

In this study, we use the functional-structural plant model SimRoot to identify phenotyping metrics that are

(i) Sensitive enough to provide information on the constituent root phenes and their states

(ii) Stable over time and are independent of the time of phenotyping

(iii) Robust to the imaging method, i.e., do not vary when measured in the intact $3 \mathrm{D}$ root system or when estimated using $2 \mathrm{D}$ rotational image series

Our analysis shows that

(i) Phene aggregates can be explained by phenes. Different phene aggregates capture different combinations of subtending phenes. However, these metrics do not provide precise information or measures of subtending phene states

(ii) Several combinations of phenes in different states can produce phenotypes which have comparable estimates of phene aggregates. Estimates of phene aggregates are not unique representations of the state of the underlying phenes

(iii) As the number of phenes captured by an aggregate phenotypic metric increases, the stability of that metric becomes less stable over time

\section{Materials and Methods}

2.1. Simulation of Phenotypes. The functional-structural plant model SimRoot [35] was used to simulate bean (Phaseolus vulgaris) and maize (Zea mays) root phenotypes. In SimRoot, simulated root system comprises of roots of distinct classes as specified by their root diameters, lateral root branching density, root growth rate, and root growth angle in the input parameters. The root growth angle over time depends on the gravitropism. Stochasticity is included in all parameters. The roots are simulated as small connected root segments over time. Coordinates corresponding to the root being simulated as well as the root length, volume, and area parameters are stored for the simulated root segments as the root grows at specified time points. The root length, area, and volume of the root system are estimated by integrating the respective parameters over all root segments. The root image coordinates are used to visualize the simulated root system. Plastic responses to local environmental variables were not considered in this study.

The number of roots of different root classes, angle, diameter, and lateral root branching density (LRBD) was varied to produce 1500 maize root phenotypes and 1500 bean root phenotypes. The range of values used for each of the root parameter used is given in Supplementary Material 2. 


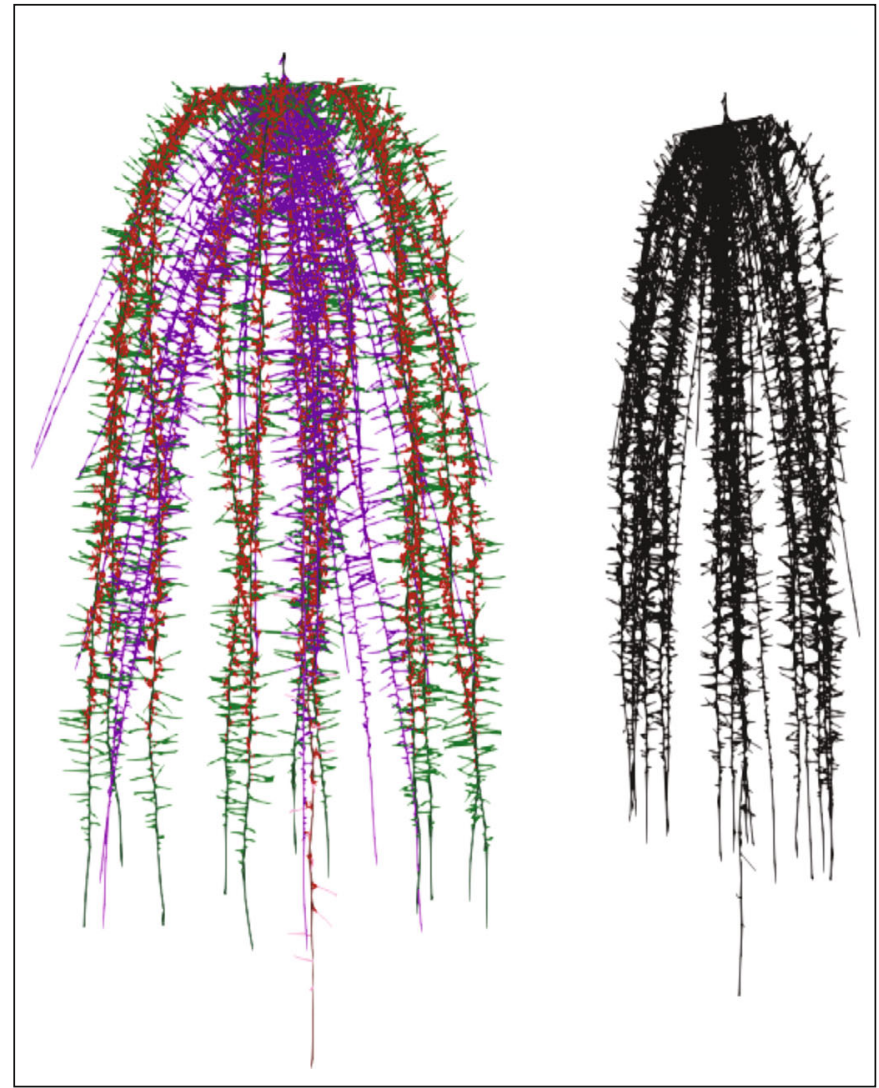

(a)

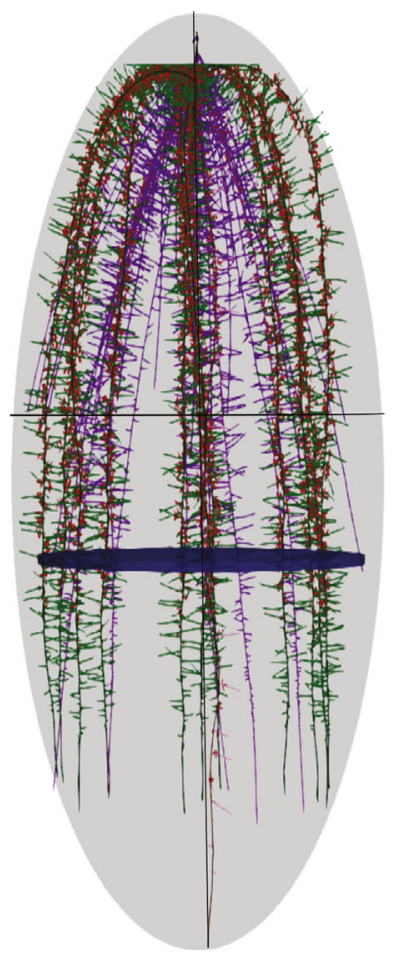

(b)

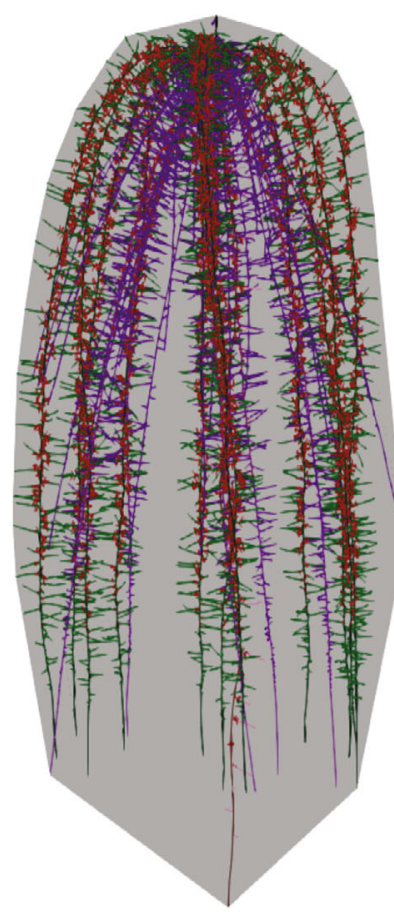

(c)

Figure 1: Representation of 2D projection of a 3D root system (a), visualization of maximum width, major ellipse axis of a 2D root system (b), and convex hull volume of a $3 \mathrm{D}$ root system (c).

The data corresponding to the simulated root phenotypes were saved during the simulation runs. These data files contained the $X, Y$, and $Z$ coordinates of the simulated root system images used to simulate the root as well as data of root length, area, volume, etc., of the simulated root segments with their corresponding root class. Roots were allowed to grow without any boundaries so that the growing roots did not touch any boundary surface, and so no artifacts were introduced due to mirroring roots. Root growth angle was influenced by root gravitropism. The angle made by the root at $5 \mathrm{~cm}$ with the horizontal (soil surface) was calculated from the image coordinates and used as estimate of root angle. In order to obtain accurate estimates of all the phenotypic traits, elementary and aggregate phenotypic were extracted/calculated from the data of the simulated images.

2.2. Measurement of Phene and Aggregate Phene Metrics. Estimates of phene metrics were measured from the simulated images. Aggregate phenotypic trait metrics were calculated for intact 3D root systems as well as projections of the root systems on a $2 \mathrm{D}$ plane. The root system was rotated by 20 degrees, and the projections on a 2D plane were obtained (Figure 1 and Supplementary Figure S1). The average of the estimates of each metric in all the projected images for each phenotype was used in studies considering 2D projections. The average value was used also in $3 \mathrm{D}$ studies where $3 \mathrm{D}$ estimates were not obtained including ellipse major axis, ellipse minor axis, and ellipse aspect ratio. The phene aggregates estimated and considered in this study, the definitions of these traits, and the method of obtaining those metrics from SimRoot output are given in Table 1. In order to evaluate how phene metrics and phenotypic trait metrics change over time, root images were obtained every 5 days starting 10 days after germination and metrics obtained for these root systems. This way phenotyping metrics were obtained for 3D root systems, 2D projections of the root systems, and root system images after different periods of growth.

2.3. Random Forest Analysis. Data obtained from 3D root systems were analyzed using random forest regression. For metrics where 3D metric data were not available (ellipse minor axis, ellipse major axis, and ellipse aspect ratio), the average value of the aggregate phenotypic trait from $2 \mathrm{D}$ rotational series was used. Random forest is a nonparametric technique derived from classification and regression trees (CART). Random forest consists of a combination of many trees, where each tree is generated by boot-strap samples, leaving about a third of the overall sample for validation (the out-of-bag predictions (OOB)). Each split of the tree is determined using a randomized subset of the predictors at each node. The final outcome is the average of the results of all the trees $[36,37]$. It uses the OOB samples (independent observations from those used to grow the tree) to calculate 
TABle 1: Aggregate phene metrics, definition, and method of obtaining them from SimRoot output.

\begin{tabular}{|c|c|c|c|c|}
\hline Parameter & $3 \mathrm{D}$ & $2 \mathrm{D}$ & Description & Measurement \\
\hline Total length & $\mathrm{Y}$ & $\mathrm{Y}$ & Summed length along the whole root system & Calculated from SimRoot output \\
\hline Total area & $\mathrm{Y}$ & $\mathrm{Y}$ & Summed surface area of the whole root system & Calculated from SimRoot output \\
\hline Total volume & $\mathrm{Y}$ & $\mathrm{Y}$ & Summed volume of the whole root system & Calculated from SimRoot output \\
\hline Maximum width & $\mathrm{Y}$ & $\mathrm{Y}$ & Maximum horizontal width of the whole root system & $\begin{array}{l}\text { Calculated using minimum enclosing } \\
\text { circle algorithm in } R\end{array}$ \\
\hline Maximum depth & $\mathrm{Y}$ & $\mathrm{Y}$ & Maximum vertical depth of the whole root system & Calculated from SimRoot output \\
\hline Median no. of roots & $\mathrm{Y}$ & $\mathrm{Y}$ & Median no. of roots from root counts & Calculated from SimRoot output \\
\hline Maximum no. of roots & $\mathrm{Y}$ & $\mathrm{Y}$ & $\begin{array}{l}\text { No. of roots at the 84th percentile of a sorted list (smallest to } \\
\text { largest) of root counts }\end{array}$ & Calculated from SimRoot output \\
\hline Bushiness & $\mathrm{Y}$ & $\mathrm{Y}$ & Ratio of the maximum no. of roots to the median no. of roots & Calculated from SimRoot output \\
\hline Volume distribution & $\mathrm{Y}$ & $\mathrm{Y}$ & $\begin{array}{l}\text { Ratio of the volume of the root system contained above one- } \\
\text { third depth of the root system to the volume of the root } \\
\text { system contained below one-third depth of the root system }\end{array}$ & Calculated from SimRoot output \\
\hline Convex hull volume & $\mathrm{Y}$ & $\mathrm{Y}$ & $\begin{array}{l}\text { Volume of the convex hull that encompasses the whole root } \\
\text { system }\end{array}$ & Obtained using Convhulln $\mathrm{f}$ \\
\hline Convex hull area & $\mathrm{Y}$ & $\mathrm{Y}$ & $\begin{array}{l}\text { Surface area of the convex hu } \\
\text { roots }\end{array}$ & Obtained using Convhulln fun \\
\hline Solidity & $\mathrm{Y}$ & $\mathrm{Y}$ & Ratio of volume to convex hull volume & Calc \\
\hline Major ellipse axes & $\mathrm{Y}$ & $\mathrm{N}$ & $\begin{array}{l}\text { Length of major axis of an ellipse best fit to overall shape and } \\
\text { size of root system }\end{array}$ & $\begin{array}{l}\text { Obtained using minimum volume } \\
\text { enclosing ellipse algorithm in } R\end{array}$ \\
\hline Minor ellipse axes & $\mathrm{Y}$ & $\mathrm{N}$ & $\begin{array}{l}\text { Length of minor axis of an ellipse best fit to overall shape and } \\
\text { size of root system }\end{array}$ & $\begin{array}{l}\text { Obtained using minimum volume } \\
\text { enclosing ellipse algorithm in } R\end{array}$ \\
\hline Ellipse axis aspect ratio & $\mathrm{Y}$ & $\mathrm{N}$ & Ratio of major axis of ellipse to minor axis & $\begin{array}{l}\text { Calculated from minor ellipse axes and } \\
\text { major ellipse axes }\end{array}$ \\
\hline Fractal dimension (FD) & $\mathrm{Y}$ & $\mathrm{Y}$ & $\begin{array}{l}\text { Measure of root complexity. Fractal dimension expresses the } \\
\text { space filling properties of a structure (e.g., root system) and is } \\
\text { associated with branching pattern }\end{array}$ & $\begin{array}{l}\text { Obtained using box count code written } \\
\text { in } R\end{array}$ \\
\hline Fractal abundance (FA) & Y & $\mathrm{Y}$ & $\begin{array}{l}\text { Measure of root complexity. Fractal abundance is associated } \\
\text { with the volume of space explored }\end{array}$ & $\begin{array}{l}\text { Obtained using box count code written } \\
\text { in } R\end{array}$ \\
\hline
\end{tabular}

error rates and variable importance; no test data or crossvalidation is required. However, this method does not calculate regression coefficients nor confidence intervals [37]. It allows the computation of variable importance measures that can be compared to other regression techniques. The $\mathrm{R}$ package random forest was employed for the data analyses, with ntree $=1000$ and mtry $=8$. Random forest regression was used with each aggregate phenotypic metric as the dependent variable and the input variables as the independent variables to identify the most important variables. The selection of the most relevant variables to include in the final model was done by ranking the variables according to their importance and excluding the least important variables. The variable importance measure based on the mean decrease in accuracy was used for selecting the important variables. Variable importance is measured by mean squared error of a variable $p$, which is averaged increase in prediction error among all regression trees when the $\mathrm{OOB}$ data for variable $p$ is randomly permuted. If variable $p$ is important, there will be an increase in prediction error. Random forest was conducted 50 times and 90 percentile from distribution of mean squared error as the significance threshold of individual variables. The variables thus chosen were used to run a reduced variable model of the original random forest model for each aggregate metric. The reduced variable models were deemed acceptable if the random forest trained upon the most important descriptors gave a fit to the data set which was similar or better than that trained upon all variables.

2.4. Variation in Estimates of Phene Aggregate Metrics. One aspect of the study was to find if estimates of aggregate phenotypes were a unique representation of the phenes. To address this, a representative phenotype was chosen for the maize root system, and phenotypes varying by less than $1 \%$ of an aggregate phenotypic trait, a shape phenotypic trait (convex hull volume) and a complex phenotypic trait (FD), were chosen to find if the phenes constituting the phenotype varied when the aggregate phenotypic trait was similar. In an alternate approach, the estimates of convex hull volume and FD of bean root phenotypes with differences in basal root whorl number and root growth angles with distinct functional value [38] were studied.

2.5. Estimates of Phene and Aggregate Phene Metrics Obtained from 2D Projections. In order to study the variation in metrics estimated in $2 \mathrm{D}$ rotational image series, the coefficient of variation for each phenotype for each phenotypic 
trait metric was calculated from $2 \mathrm{D}$ projections of the root system, and the phenotypic metrics were compared.

2.6. Estimates of Phene and Aggregate Phene Metrics over Time. Root system image data were saved every 5 days from day 10 to day 40 of growth, and the $3 \mathrm{D}$ estimates of the phenes and phene aggregates were collected.

\section{Results}

Different bean and maize phenotypes were simulated by varying input parameters in SimRoot.

3.1. Variation in Simulated Phenotypes. The estimates of all phenotypes were min-max scaled, and the phenotypes were clustered by hierarchical cluster analysis of the phenotypes based on their phenes. The results of our study are based on a wide array of phenotypes. Phenotypes included in the study had vastly different phenotypes and differed in few or many phenes. The heat map in Figure 2(a) shows a small subset of data: the relative values of the bean phenes in a few phenotypes (rows) and the corresponding phenotypes in Figure 2(b). Phenotype 1 had very shallow basal root growth angle compared to phenotype 2 while phenotypes 8 and 9 had deep basal root growth angles. Phenotype 7 had more basal roots than the other phenotypes. Phenotypes 5 and 6 differed in the basal root branching density as well as basal root angle. The heat map in Figure 3(a) shows a small subset of data: the relative values of maize phenes in a few phenotypes (rows) and the corresponding phenotypes in Figure 3(b). Phenotypes 2 and 3 differed in the number of nodal roots with phenotype 2 having more nodal roots than phenotype 3 . Phenotypes 4 and 6 had similar primary root lateral branching, but phenotype 6 had no seminal roots while phenotypes 4 had 5 seminal roots. Phenotypes 8 and 9 differed in the number of seminal roots as well as seminal root LRBD and the number of nodal roots. The heat map of all bean root phenotypes and representative phenotypes considered in this study is included in Supplementary Figure S2(a) and S2(b). A similar heat map for maize root phenotypes is presented in Supplementary Figure S3(a) and S3(b), respectively.

3.2. Correlation among Phenotypic Metrics. Strong correlations were found among the phenes (Figures 4(a) and 4(b)), in the bean root system as well as the maize root system. Axial root length was negatively correlated with diameter, number, and LRBD of basal roots in bean and nodal roots in maize root system. The primary axial root length and seminal axial root length were negatively correlated with diameter of the primary root; seminal root axial root length was also negatively correlated with nodal root LRBD. Phenotypes with longer axial roots had greater maximum width, maximum depth, convex hull area, convex hull volume, major ellipse axis, and minor ellipse axis but smaller values for solidity (Figure 4(b)). Solidity was positively correlated with diameter and number of basal roots in bean. Strong correlations also exist between aggregate phenotypic trait metrics. Major ellipse axis positively correlated with maximum depth. Convex hull area, convex hull volume, minor ellipse axis, and maximum width are highly positively correlated with each other but are negatively correlated with solidity (Figure 4).

3.3. Random Forest Analysis: Different Phenes Are Important in Determining the Estimate of Different Aggregate Phenes. The results of the random forest analysis are shown in Table 2. Reduced variable models created with random forest show proportion of explained variance $\left(R^{2}\right)$ between $80 \%$ and $99 \%$ for models with all aggregate phenotypic metric except bushiness, which had $62 \%$ in bean and $41 \%$ in maize, and $F D$, which had $R^{2}$ of $67 \%$ in bean and $20 \%$ in maize. The most important variables for each aggregate phenotype for the bean and maize models are summarized in Table 3 . The variables have been summarized based on the phene the variable represents.

Among the variables evaluated by the random forest analysis, axial root length and lateral root length were found to be important explanatory variables for all the phene aggregates in both bean as well as maize. Lateral root branching density (LRBD) was found to be an important variable for total length, total area, total volume, maximum number of roots, median number of roots, bushiness, FD, and FA in bean as well as maize. LRBD was also important for volume distribution in maize root phenotypes and ellipse aspect ratio in bean root phenotypes. Number of roots and diameter played important roles in determining the total area in maize and bean root systems, respectively. Root diameter was an important variable for total volume, volume distribution, maximum depth, solidity, and FD in both bean and maize phenotypes. Diameter was also an important variable in total area and ellipse aspect ratio in bean and bushiness in maize root phenotypes. Angle was selected as an important variable by the random forest models for maximum width, convex hull area, convex hull volume, ellipse minor axis, ellipse aspect ratio, solidity, and $F D$ for both maize and bean. All the variables evaluated are important for the model with FD as the dependent variable.

3.4. Estimates of Aggregate Phene Metrics Can Be Similar for Phenotypes with Different Phene States. Even in phenotypes with similar estimates for aggregate phenotypic metrics, the phene states of the constituent phenes varied greatly (Figures 5(a) and 5(b)). Phenotypes chosen based on the similarity of aggregate phenotypic metrics had different diameter, LRBD, and number of roots of different classes. Conversely, phenotypes in which phenes exist in different states have similar aggregate phenotypic metrics (Figure 6). Four bean phenotypes that vary only in the number of basal roots and root growth angle were chosen, and the estimate of total volume, convex hull volume, and FD were compared (Figure 6). Phenotype 1 has one whorl of basal roots with shallow angles, phenotype 2 has one whorl of basal roots with deep angles, and phenotype 3 has three whorls with fanned root growth angles. While phenotypes 1 and 2, which vary only in root growth angle, have different estimates for all the three metrics considered (total volume, convex hull volume, and FD), phenotypes 1 and 3 have similar estimates for FD (varying by less than $2 \%$ ) even though they vary in both in number of basal roots as well as root growth angles. 


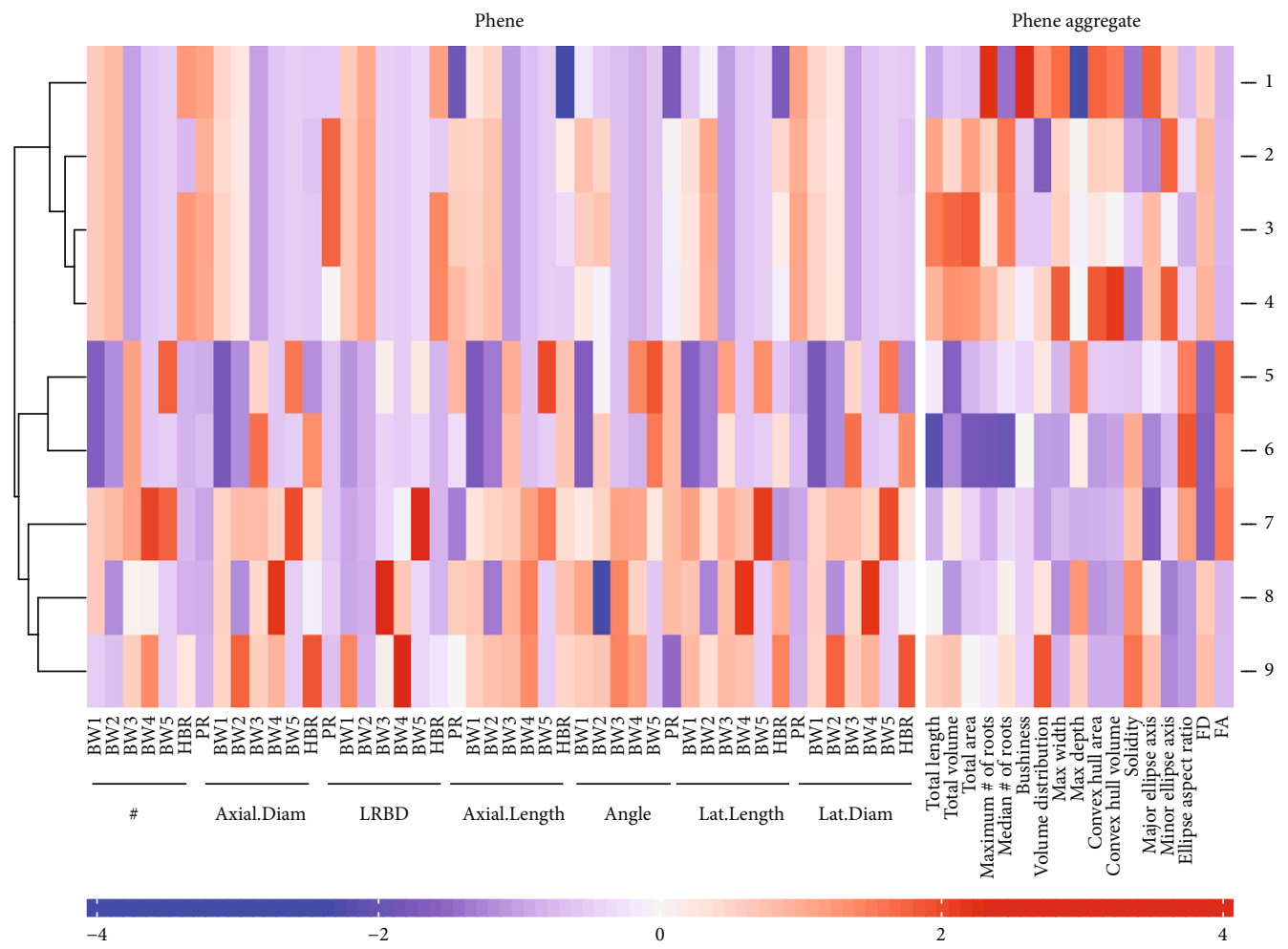

(a)
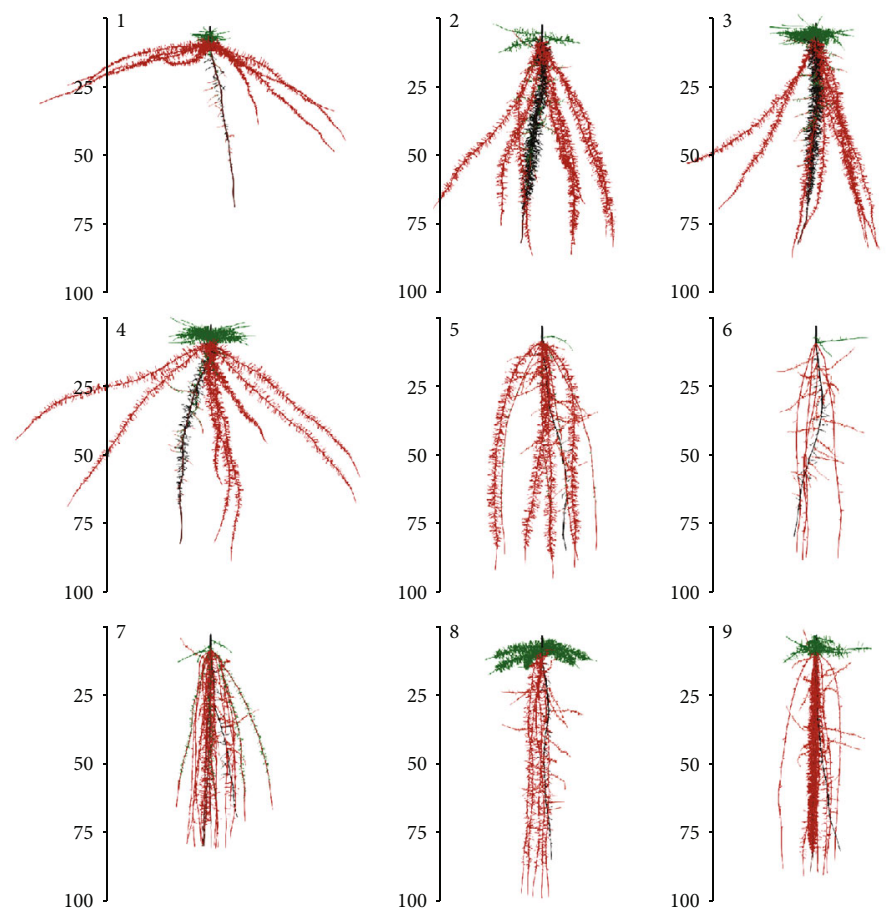

(b)

Figure 2: Cluster heat map of phenotypic traits. Hierarchical clustering of a few phenotypes was generated using Spearman correlation of max-min scaled phene values of bean phenotypes at 40 days (a). The color scale indicates the magnitude of the trait values (blue, low value; red, high value). The numbers indicated on the heat map refer to the phenotype in the specific row of the heat map. The corresponding phenotypes are visualized in (b). Primary roots are in black; basal roots are in red; hypocotyl-borne roots are in green. \#: number of axial roots; Axial.Diam: axial root diameter; LRBD: lateral root branching density; Lat.Length: lateral root length; Lat.Diam: lateral root diameter; BW1: basal roots at whorl 1; BW2: basal roots at whorl 2; BW3: basal roots at whorl 3; BW4: basal roots at whorl 4; BW5: basal roots at whorl 5; HBR: hypocotyl-borne roots; PR: primary root. 


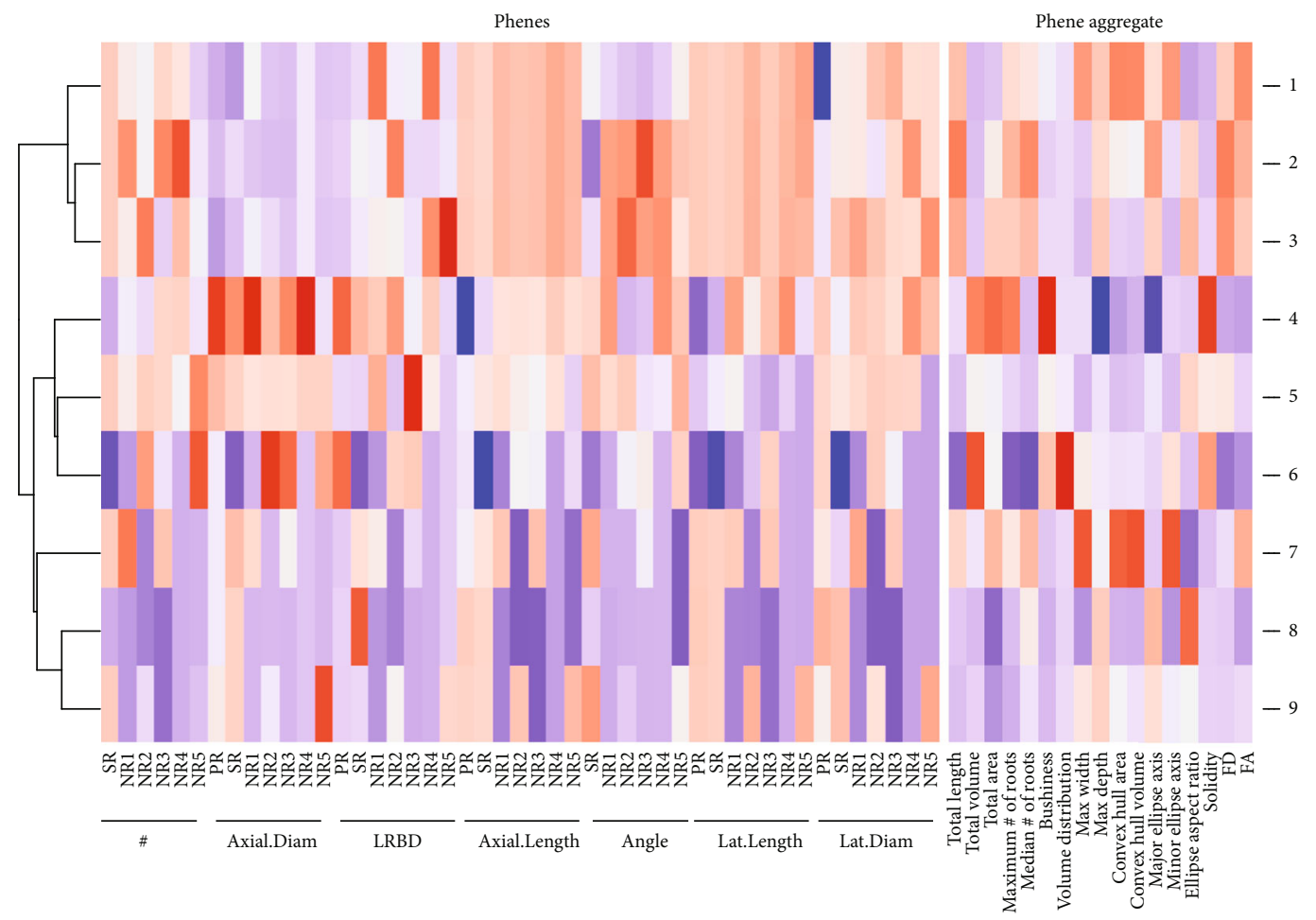

(a)
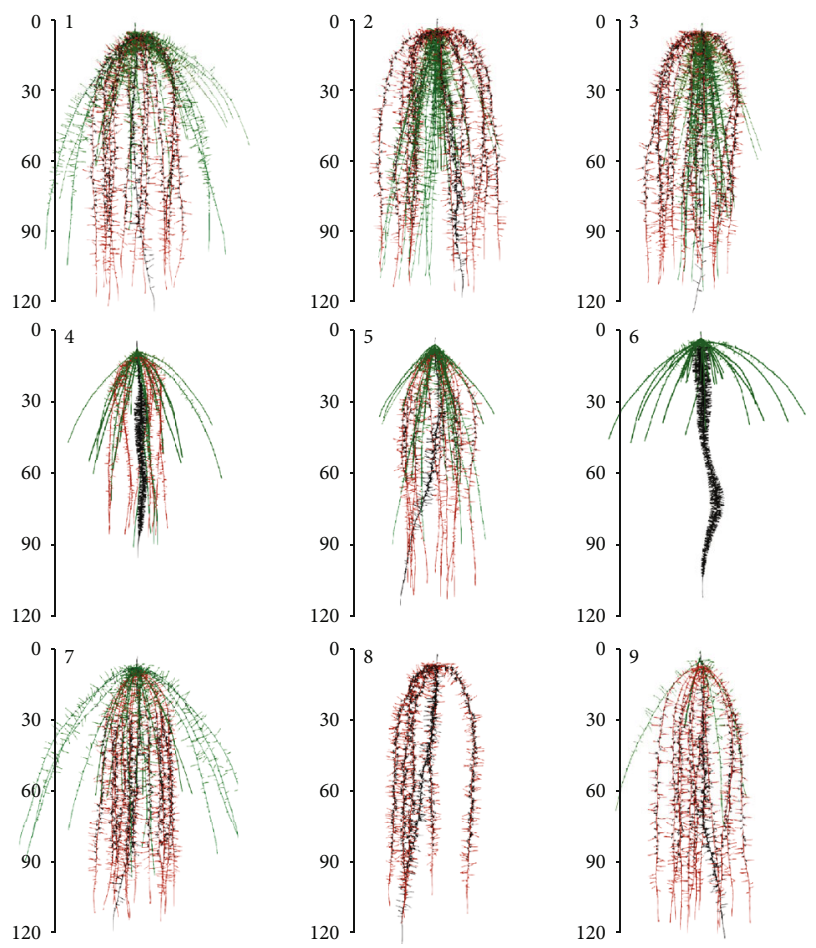

(b)

Figure 3: Cluster heat map of phenotypic traits. Hierarchical clustering of a few phenotypes was generated using Spearman correlation of max-min scaled phene values of maize phenotypes at 40 days (a). The color scale indicates the magnitude of the trait values (blue, low value; red, high value). The numbers indicated on the heat map refer to a phenotype in the specific row of the heat map. The corresponding phenotypes are visualized in (b). Primary roots are in black; seminal roots are in red; nodal roots are in green. \#: number of axial roots; Axial.Diam: axial root diameter; LRBD: lateral root branching density; Lat.Length: lateral root length; Lat.Diam: lateral root diameter; NR1: nodal roots at position 1; NR2: nodal roots at position 2; NR3: nodal roots at position 3; NR4: nodal roots at position 4; SR: seminal roots; PR: primary root. 


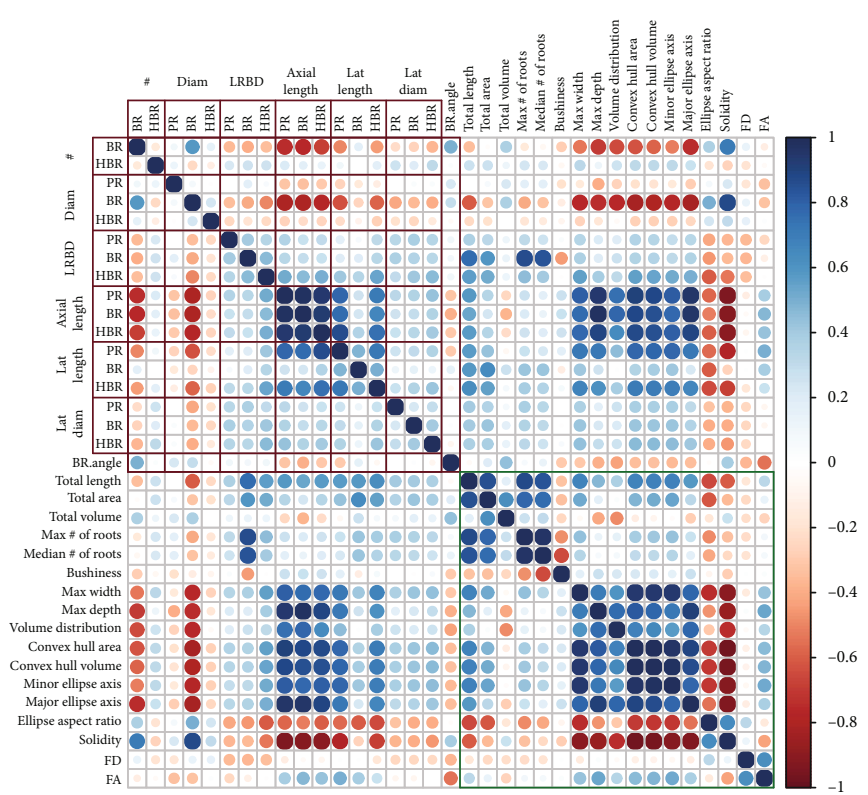

(a)

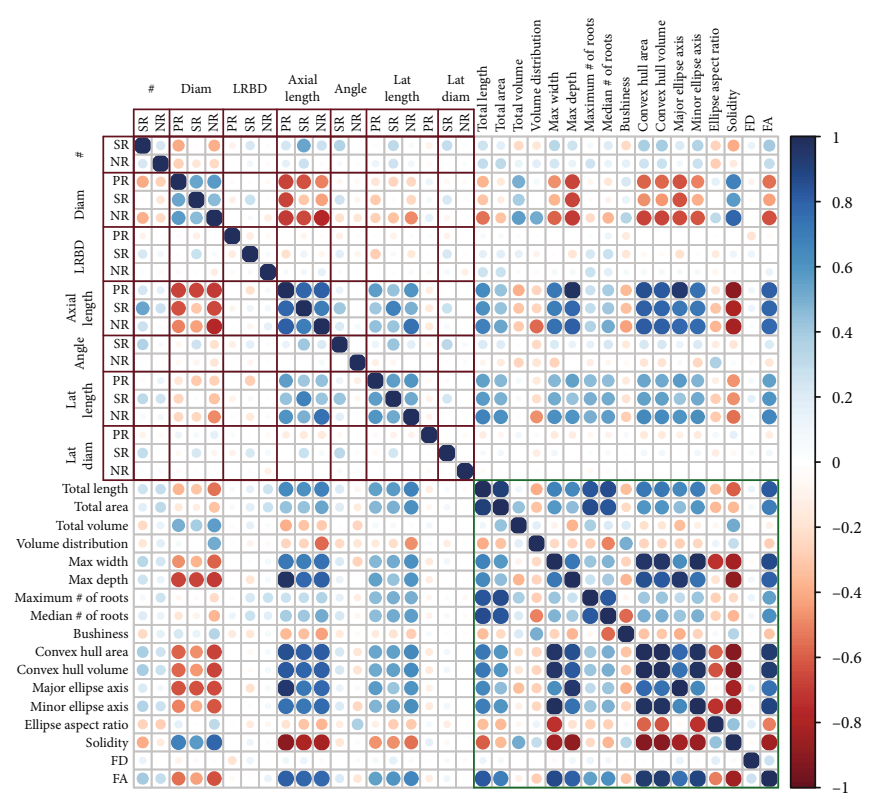

(b)

Figure 4: Phenotypic trait relationship. Correlation matrix of phenes and phene aggregates evaluated for bean root phenotypes (a). Correlation matrix of phenes and phene aggregates evaluated for maize root phenotypes (b). The color scale indicates Spearman correlation coefficient between traits (red, negative; blue, positive). Color intensity and size of the circle are proportional to the correlation coefficients between two traits. Correlations between phenes are indicated by the points in the red box; the green box contains the correlations between phene aggregates. BR: basal roots; HBR: hypocotyl-borne roots; PR: primary root; SR: seminal roots; NR: nodal roots; \#: number of axial roots; Diam: axial root diameter; LRBD: lateral root branching density; Axial Length: axial root length; Lat Length: lateral root length; Lat Diam: lateral root diameter.

Similarly, phenotype 4 has four whorls with fanned angles and differs from phenotype 3 and phenotype 1 in number of basal roots as well as root growth angle but varies in the estimates of total volume by $1 \%$ and $16 \%$, respectively, and in the estimate of convex hull volume by $1 \%$ and $4 \%$, respectively (Figure 6).

3.5. Variation in Estimates of Phene and Phene Aggregate Metrics Obtained from 2D Projections. In order to study which metrics are not accurately represented by $2 \mathrm{D}$ projections, elementary and aggregate phenotypic metrics were estimated from $2 \mathrm{D}$ projections obtained by rotating the root system through 360 degrees at 20-degree intervals. It should be noted that convex hull volume and area of a $2 \mathrm{D}$ projection corresponds to surface area of a 2D hull and the length of the perimeter of a 2D hull, respectively. Analysis with 2D image series shows that among phenes, estimates of root growth angle differ when projections are obtained at different rotations. Among aggregate phenotypic trait metrics, the metrics which have angle as one of the most important variables, including convex hull volume, convex hull area, minor ellipse axis, major ellipse axis, ellipse aspect ratio, solidity, FD, and $F A$, as determined in the random forest analysis, are sensitive to projection. These phenotypic metrics had a coefficient of variation of $10-20 \%$, but some had much greater CV depending on the phenotype in both maize and bean (Figures $7(\mathrm{a})$ and $7(\mathrm{~b})$ ). The differences in estimates inflated when an aggregate phenotypic trait was calculated as a function of two metrics which are already subject to lot of measurement variation (Figures $7(\mathrm{a})$ and $7(\mathrm{~b})$ ).
3.6. Variation in Estimates of Phene and Phene Aggregate Metrics over Time. Some phene aggregates increase substantially over 30 days, while some remained relatively static and estimates of some aggregate metric decreased with time (Figures 8(b) and 9(b)). Of the traits, total length, total area, total volume, maximum depth, convex hull area, convex hull volume, major ellipse axis, minor ellipse axis, and FA progressively increased over time in both bean and maize (Figures 8(b) and 9(b), Supplementary Figure S4(b), Supplementary Figure S5(b)). There was only a small change in the maximum number of roots in bean over time, but this value increased significantly in maize over time (Supplementary Figure S5(b)). The pattern of changes in $F D$ over time was dependent on the phenotype. There was a small decrease in bushiness of bean over time (Figure 8(b)). In maize, the phenotypes showed a significant increase from day 10 to 20 followed by a drop from day 20 onwards (Figure 9(b)). The magnitude of increase was dependent on the phenotype. Volume distribution was either static, or there was a slight increase in the bean phenotypes over time (Supplementary Figure S4(b), Supplementary Figure S5(b)). In maize, the change in magnitude of volume distribution over time was dependent on the phenotype.

\section{Discussion}

This study investigated the importance and utility of phenes and phene aggregate traits in phenotyping studies. Our results confirm that phenes are robust and stable over time and also sensitive enough to discriminate between highly 
TABLE 2: Results of regression models created with random forest. The $R^{2}$ values of random forest model with entire set of variables and those with only most important variables are presented for the bean and maize aggregate phene metrics.

\begin{tabular}{|c|c|c|c|c|}
\hline \multirow{3}{*}{ Aggregate phenotypic metric } & \multicolumn{4}{|c|}{$R^{2}$ (\% variance explained) } \\
\hline & \multicolumn{2}{|c|}{ Bean } & \multicolumn{2}{|c|}{ Maize } \\
\hline & Model with all variables & $\begin{array}{l}\text { Model with most } \\
\text { important variables }\end{array}$ & Model with all variables & $\begin{array}{l}\text { Model with most } \\
\text { important variables }\end{array}$ \\
\hline Total length & 89.5 & 91.6 & 82 & 85 \\
\hline Total area & 87 & 87 & 78 & 81 \\
\hline Total volume & 81.7 & 88.5 & 79 & 81.6 \\
\hline Volume distribution & 87 & 91 & 61 & 66 \\
\hline Max no. of roots & 78.8 & 84 & 67 & 72.8 \\
\hline Median no. of roots & 79.9 & 87 & 71 & 75 \\
\hline Bushiness & 62 & 67 & 36 & 41 \\
\hline Max depth & 98.6 & 99.6 & 79 & 84 \\
\hline Max width & 91 & 90 & 95 & 99 \\
\hline Convex hull area & 97.8 & 97 & 90 & 93.4 \\
\hline Convex hull volume & 97.6 & 97.6 & 87 & 89.9 \\
\hline Ellipse minor axis & 94.9 & 93.6 & 80 & 85 \\
\hline Ellipse major axis & 96.7 & 97.3 & 95 & 98.6 \\
\hline Ellipse aspect ratio & 85.9 & 87.4 & 51.9 & 62 \\
\hline Solidity & 97.4 & 97.5 & 89 & 89 \\
\hline FD & 67 & 68 & 16 & 20 \\
\hline FA & 93.5 & 94.9 & 88 & 90 \\
\hline
\end{tabular}

Random forest possesses its own reliable statistical characteristics, which could be used for validation and model selection. The major criterion for estimation of internal predictive ability of the random forest models and model selection is the value of $R^{2} . R^{2}$ in random forest is interpreted as a measure of predictive quality of random forest model on independent samples. Random forest models were run with the aggregate phenotype as dependent variable and all the phenes as predictor variables. Most important variables were chosen based on the $\%$ increase in mean square, and random forest models were run with only the most important variables.

similar root systems. In contrast, since phene aggregates capture combinations of subtending phenes and several combinations of phenes in different states can produce phenotypes which have comparable estimates of phene aggregates, the estimates of phene aggregates are not unique representations of the state of the underlying phenes. Aggregate phene metrics are not stable over time, mostly because there is a rapid development of many elementary root phenes over time. When the number of phenes estimated by the aggregate metric increases, the complex interactions among phenes result in the same phenotype having vastly different estimates for the same aggregate metric at different time points.

4.1. Root Models Can Aid Exploration of Root Phenomics. In this study, we use SimRoot to simulate root systems and use the simulated phenotypes to evaluate various root phenotyping metrics. We used modelling for this study due to constraints in obtaining empirical data caused by limitations in phenotyping methodologies and artifacts due to technicalities in image processing. Phenotyping efforts represent a compromise between throughput, precision, and data processing. Many high-throughput phenotyping methodologies involve obtaining $2 \mathrm{D}$ metrics and depend on growing plants in controlled growth systems such as pouch, pots, gel plate systems, and germination paper where root architecture is affected due to spatial growth constraints, in particular, branching angles. Not all 3D RSA estimates can be obtained by series of $2 \mathrm{D}$ image data; some phenotyping metrics such as volume of nonconvex shapes cannot be obtained from $2 \mathrm{D}$ projections, especially from complex root systems. Occlusions in $2 \mathrm{D}$ images caused by crossing roots increase complexity of systems and reduce accuracy of many 2D estimates; this is especially true for mature root systems which are complex branched structures composed of overlapping and crossing segments [39]; 3D estimates are better for measuring these "traits" but are biased for other parameters such as surface area due to technicalities in image reconstructions. 3D imaging techniques such as X-ray computed tomography $(\mu \mathrm{CT})$ and magnetic resonance imaging allow noninvasive studying of spatiotemporal dynamics of root growth [40-43] but require elaborate data processing and are suitable for relatively small and young root systems due to technical restrictions in container size $[44,45]$ and are scanned at low throughput $[45,46]$. Studies under controlled conditions enable study of growth of roots over time however are generally used to assess less complex root structures on younger plants from germination to ca. 10 day after germination [15]. This is a particular limitation for monocot roots which develop more axial roots over time. Destructive field sampling methods such as shovelomics [25, 47] allow the measurement of the root crown phenotype however is associated with loss and possible displacement of fine roots $[48,49]$. Estimates of phenotyping metrics such as 
TABLE 3: Summary of the most important variables selected by random forest model for each phenotyping metric evaluated for bean root system and maize root system.

\begin{tabular}{|c|c|c|c|c|c|c|}
\hline \multirow{2}{*}{ Phene aggregates } & \multicolumn{6}{|c|}{ Phenes } \\
\hline & Axial root length & No. of roots & LRBD & Angle & Diameter & Lateral root length \\
\hline Total length & $\begin{array}{l}\text { Maize } \\
\text { Bean }\end{array}$ & $\begin{array}{l}\text { Maize } \\
\text { Bean }\end{array}$ & $\begin{array}{l}\text { Maize } \\
\text { Bean }\end{array}$ & & & $\begin{array}{l}\text { Maize } \\
\text { Bean }\end{array}$ \\
\hline Total area & $\begin{array}{l}\text { Maize } \\
\text { Bean }\end{array}$ & Maize & $\begin{array}{l}\text { Maize } \\
\text { Bean }\end{array}$ & & Bean & $\begin{array}{l}\text { Maize } \\
\text { Bean }\end{array}$ \\
\hline Total volume & $\begin{array}{l}\text { Maize } \\
\text { Bean }\end{array}$ & $\begin{array}{c}\text { Maize } \\
\text { Bean }\end{array}$ & $\begin{array}{c}\text { Maize } \\
\text { Bean }\end{array}$ & & $\begin{array}{c}\text { Maize } \\
\text { Bean }\end{array}$ & $\begin{array}{l}\text { Maize } \\
\text { Bean }\end{array}$ \\
\hline Volume distribution & $\begin{array}{l}\text { Maize } \\
\text { Bean }\end{array}$ & $\begin{array}{l}\text { Maize } \\
\text { Bean }\end{array}$ & Maize & & $\begin{array}{l}\text { Maize } \\
\text { Bean }\end{array}$ & $\begin{array}{l}\text { Maize } \\
\text { Bean }\end{array}$ \\
\hline Max \# of roots & $\begin{array}{l}\text { Maize } \\
\text { Bean }\end{array}$ & Maize & $\begin{array}{l}\text { Maize } \\
\text { Bean }\end{array}$ & & & $\begin{array}{c}\text { Maize } \\
\text { Bean }\end{array}$ \\
\hline Median \# of roots & $\begin{array}{l}\text { Maize } \\
\text { Bean }\end{array}$ & Maize & $\begin{array}{l}\text { Maize } \\
\text { Bean }\end{array}$ & & & $\begin{array}{l}\text { Maize } \\
\text { Bean }\end{array}$ \\
\hline Bushiness & $\begin{array}{l}\text { Maize } \\
\text { Bean }\end{array}$ & Maize & $\begin{array}{c}\text { Maize } \\
\text { Bean }\end{array}$ & Bean & Maize & $\begin{array}{c}\text { Maize } \\
\text { Bean }\end{array}$ \\
\hline Max depth & $\begin{array}{l}\text { Maize } \\
\text { Bean }\end{array}$ & & & Maize & $\begin{array}{l}\text { Maize } \\
\text { Bean }\end{array}$ & $\begin{array}{l}\text { Maize } \\
\text { Bean }\end{array}$ \\
\hline Max width & $\begin{array}{l}\text { Maize } \\
\text { Bean }\end{array}$ & & & $\begin{array}{l}\text { Maize } \\
\text { Bean }\end{array}$ & & $\begin{array}{l}\text { Maize } \\
\text { Bean }\end{array}$ \\
\hline Convex hull area & $\begin{array}{l}\text { Maize } \\
\text { Bean }\end{array}$ & & & $\begin{array}{l}\text { Maize } \\
\text { Bean }\end{array}$ & & $\begin{array}{l}\text { Maize } \\
\text { Bean }\end{array}$ \\
\hline Convex hull volume & $\begin{array}{l}\text { Maize } \\
\text { Bean }\end{array}$ & & & $\begin{array}{l}\text { Maize } \\
\text { Bean }\end{array}$ & & $\begin{array}{c}\text { Maize } \\
\text { Bean }\end{array}$ \\
\hline Ellipse minor axis & $\begin{array}{l}\text { Maize } \\
\text { Bean }\end{array}$ & & & $\begin{array}{l}\text { Maize } \\
\text { Bean }\end{array}$ & & $\begin{array}{l}\text { Maize } \\
\text { Bean }\end{array}$ \\
\hline Ellipse major axis & $\begin{array}{l}\text { Maize } \\
\text { Bean }\end{array}$ & & & & & $\begin{array}{l}\text { Maize } \\
\text { Bean }\end{array}$ \\
\hline Ellipse aspect ratio & $\begin{array}{c}\text { Maize } \\
\text { Bean }\end{array}$ & $\begin{array}{l}\text { Maize } \\
\text { Bean }\end{array}$ & Bean & $\begin{array}{l}\text { Maize } \\
\text { Bean }\end{array}$ & Bean & $\begin{array}{c}\text { Maize } \\
\text { Bean }\end{array}$ \\
\hline Solidity & $\begin{array}{l}\text { Maize } \\
\text { Bean }\end{array}$ & Maize & & $\begin{array}{l}\text { Maize } \\
\text { Bean }\end{array}$ & $\begin{array}{l}\text { Maize } \\
\text { Bean }\end{array}$ & $\begin{array}{l}\text { Maize } \\
\text { Bean }\end{array}$ \\
\hline FD & $\begin{array}{l}\text { Maize } \\
\text { Bean }\end{array}$ & $\begin{array}{c}\text { Maize } \\
\text { Bean }\end{array}$ & $\begin{array}{c}\text { Maize } \\
\text { Bean }\end{array}$ & $\begin{array}{l}\text { Maize } \\
\text { Bean }\end{array}$ & $\begin{array}{l}\text { Maize } \\
\text { Bean }\end{array}$ & $\begin{array}{c}\text { Maize } \\
\text { Bean }\end{array}$ \\
\hline FA & $\begin{array}{l}\text { Maize } \\
\text { Bean }\end{array}$ & Maize & $\begin{array}{c}\text { Maize } \\
\text { Bean }\end{array}$ & Bean & & $\begin{array}{l}\text { Maize } \\
\text { Bean }\end{array}$ \\
\hline
\end{tabular}

fractal dimension are sensitive to incompleteness of the excavated root network [19, 44].

SimRoot, a functional-structural plant model, has been used extensively for elucidating the functional value of one or more phenes and to analyze phene interactions and root complexity [6, 20, 30, 38, 50-53]. Simulations with SimRoot enable comparing genotypes that vary only in the phene of interest, i.e., near-isophenic lines, which are exceedingly difficult to obtain empirically $[12,32,38]$. A significant advantage of using SimRoot is that root architecture over time is known in its entirety devoid of measurement and sampling error. Highly complex root systems can be simulated, and resulting root images can be used without any requirement of cleaning images as there is no image noise. Root image coordinates are recorded as they grow in $3 \mathrm{D}$ space, and so root phenotyping traits can be measured at any time step for any number of time steps without addi- tional effort. One of the major hurdles in phenotyping roots is that artifacts may be present so that the representation of the root system may not be accurate.

4.2. Correlation among Estimates of Phenes and Phene Aggregates Is an Emergent Property of SimRoot. Our studies with phenes and phene aggregates show that some phenes are highly correlated with each other. SimRoot is a mechanistic model and has no fixed relationships for the root architectural parameters. The phenotype is simulated based on a set of input parameters including number of roots of different root classes, root growth angles, root diameter, and lateral root branching density with some stochasticity included in each of the parameters. Due to carbon feedbacks and restricted carbon availability, not all phenotypes are simulated. The root system develops based on carbon availability as determined by availability in the seed initially. Plant 

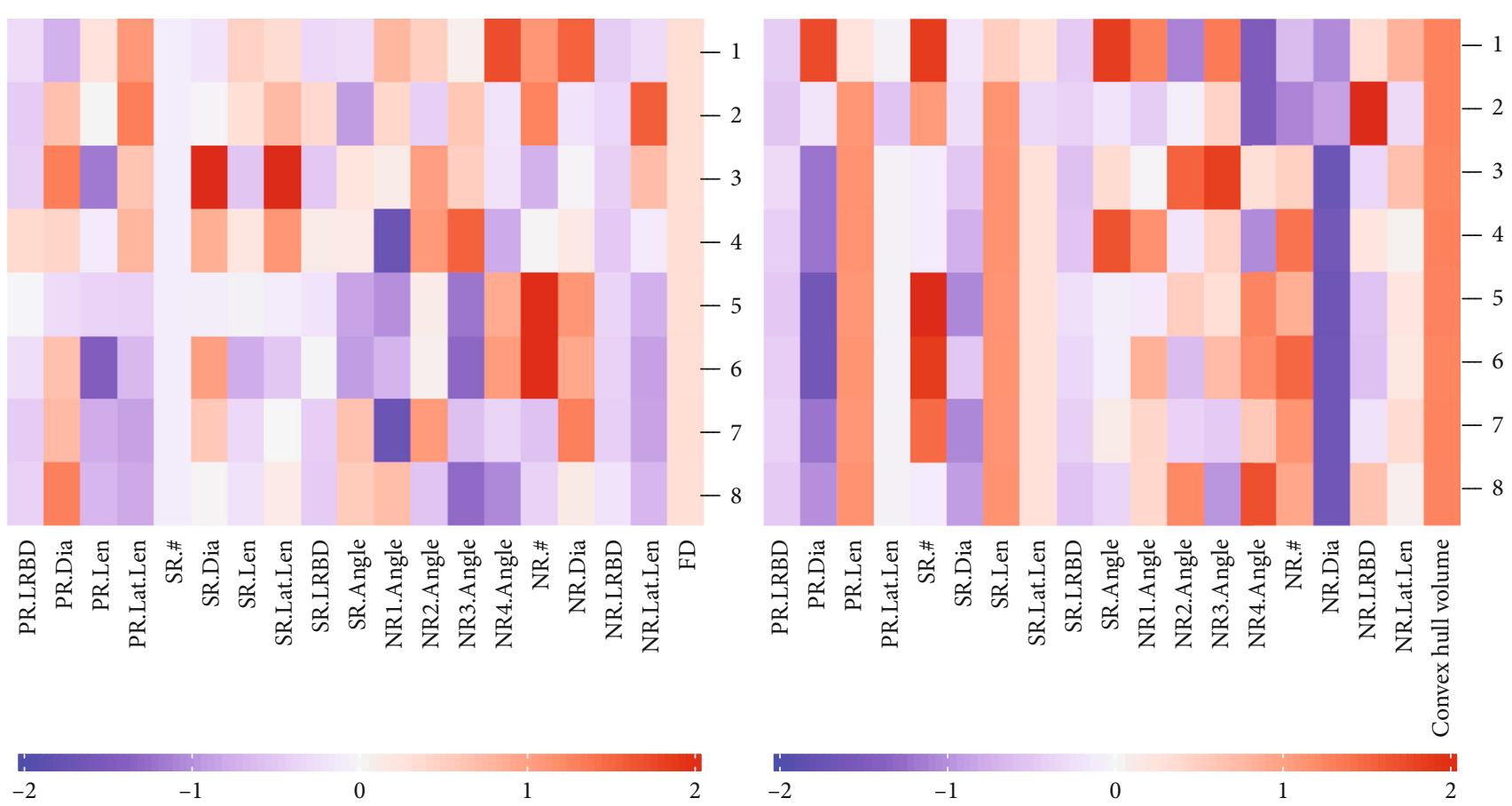

(a)

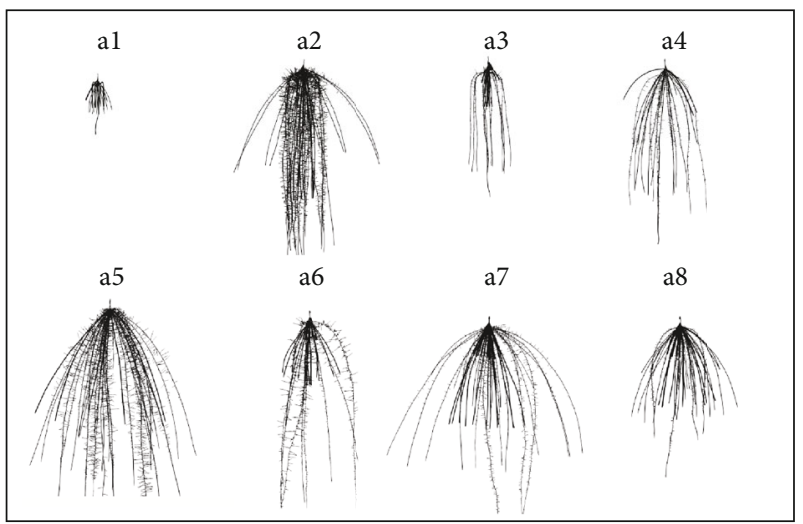

(c)

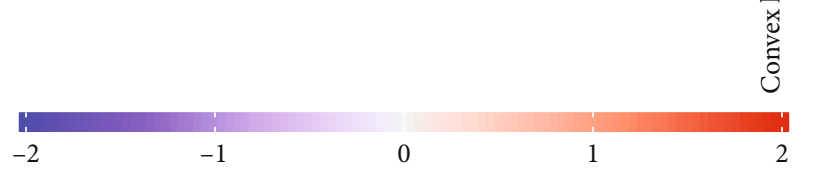

(b)

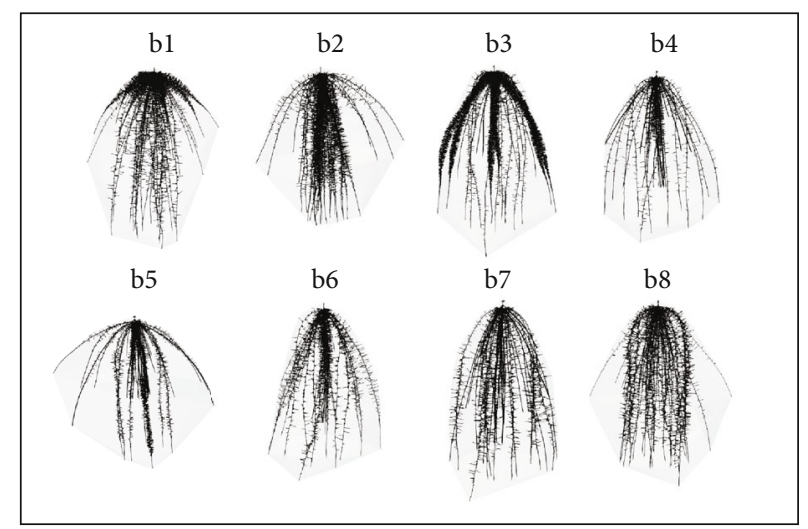

(d)

FIGURE 5: Phene values of maize root phenotypes with comparable FD (a) and convex hull volume (b). The heat map shows values of the traits obtained by dividing the values with maximum value of respective traits. The visualization of the phenotypes with similar FD and similar convex hull volume is presented in (c) and (d), respectively. Phenotypes a1-a8 have similar FD; phenotypes b1-b8 have similar convex hull volume. PR: primary root; SR: seminal root; NR: nodal root; LRBD: lateral root branching density; Len: axial root length; Lat.Len: lateral root length; \#: number of axial roots; FD: fractal dimension.

growth and development occur as emerging from underlying processes such as photosynthesis, allocation of assimilates, uptake of nutrients, and determine the growth of the plant root system $[30,38,53]$. There are no correlations built into the model, and the correlations seen among the phenes in the phenotypes are a result of the mechanistic processes that are captured in the model. For example, larger diameter root axes result in larger carbon sinks leaving few resources for other roots. A set of carbon allocation rules determines carbon allocated to different root classes with axial roots having precedence over lateral roots. This is seen as a reduction in lateral root length when the number of roots is greater or when the root diameter is greater. Growth rates of the root tips are a function of carbon availability and if severe carbon limitations occur (as would occur if the phenotype being simulated had many axial roots, greater branching density or large diameter roots, or a combination of these), axial root length is affected and in extreme cases may inhibit the emergence of roots emerging later. Attempts to factorially design phenotypes based on discrete values of the phene states resulted in some phenotypes not developing for more than few days due to carbon limitations. This is because SimRoot keeps track of resource allocation (C, N, P) and trade-offs in carbon allocation result in trade-offs among root traits, as occurs with real plants. The trade-offs include longer axial roots and longer lateral roots when number of axial 
Convex hull volume $\left(\mathrm{cm}^{3}\right)$

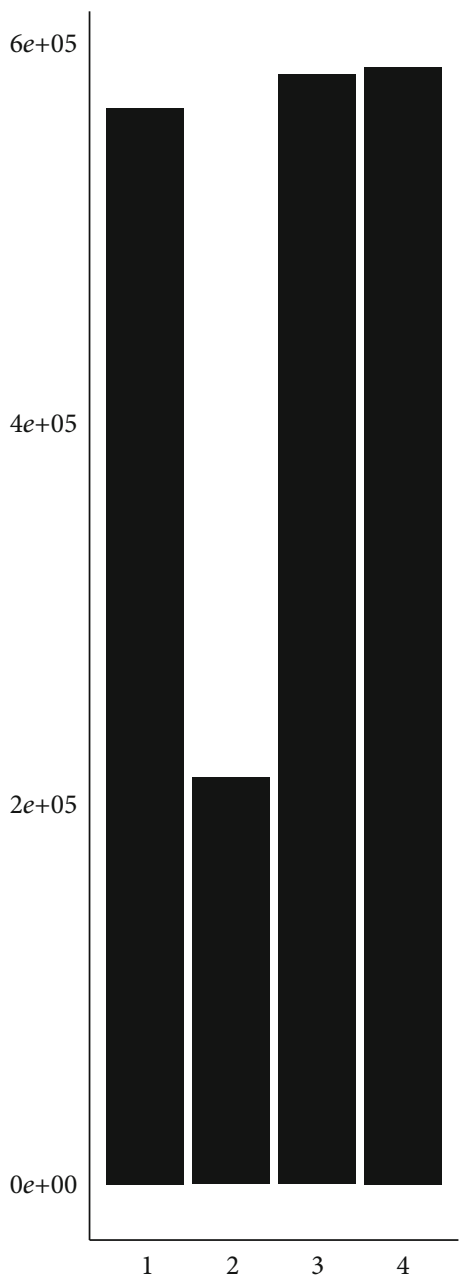

FD

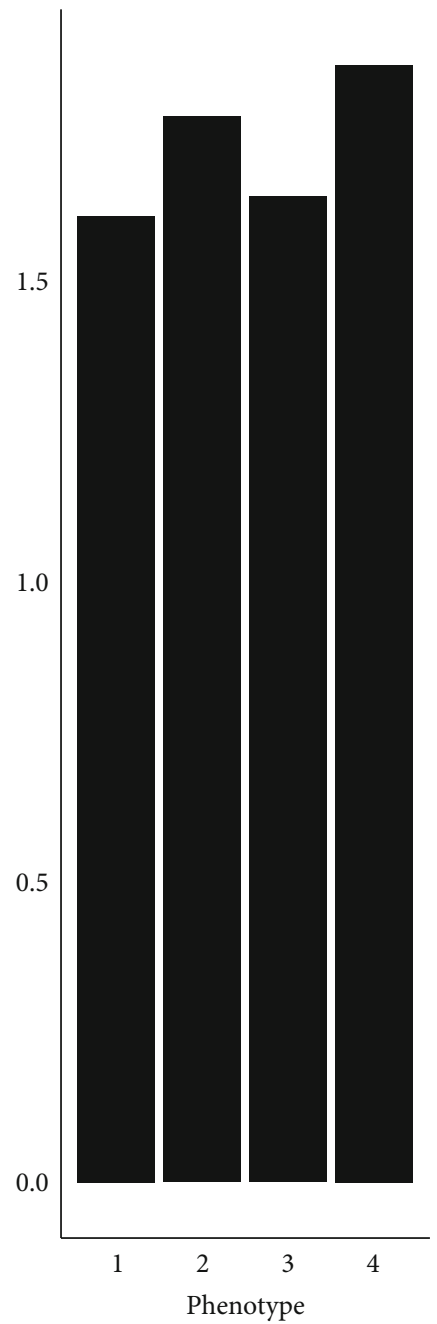

Total volume $\left(\mathrm{cm}^{3}\right)$

20

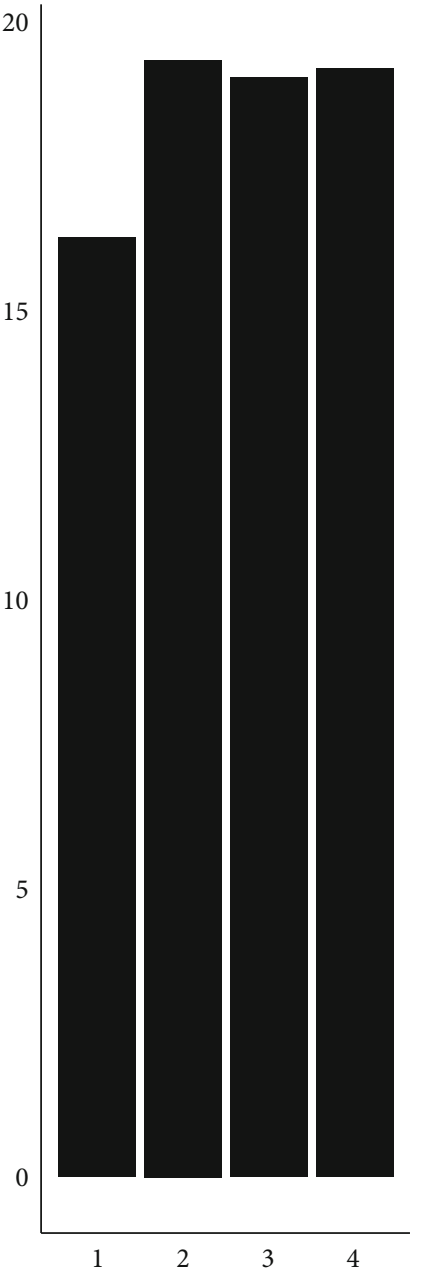

1

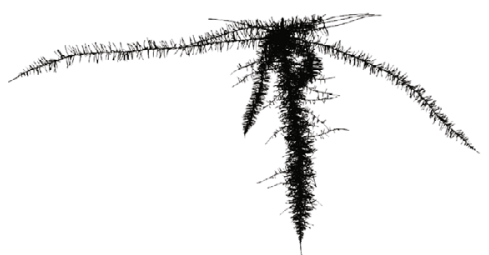

2

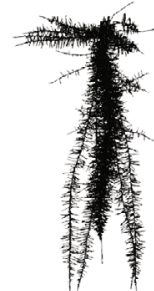

3

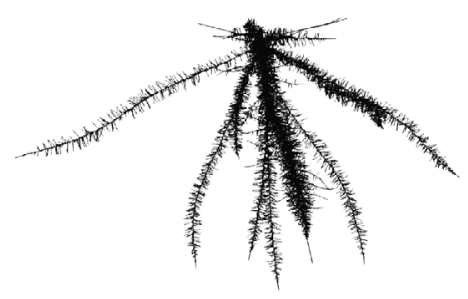

4

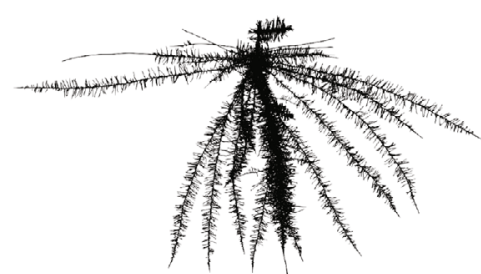

Figure 6: Convex hull volume, FD, and total volume of bean root phenotypes with (1) one whorl and shallow angle, (2) one whorl and deep angle, (3) two whorls and fanned angle, and (4) four whorls and fanned angles. The corresponding phenotypes are visualized in lower panel. FD: fractal dimension.

roots/axial root diameter is reduced, which are seen as high correlations among those phenes. Only those phenotypes that supported plant growth for 40 days were used so that the metrics were dependent only on the phenotype. All metrics were recalculated/extracted from the simulated root system in order to get an accurate estimate of the phenotypic metric.

Correlations also exist among phene aggregates; maximum depth and major ellipse axis were highly correlated; con- vex hull area, convex hull volume, maximum width, and minor ellipse axis were also highly correlated as seen in several other studies. Major ellipse axis and maximum depth are measures of rooting depth [54] and were correlated with primary root length. Maximum width, minor ellipse axis, and convex hull are phene aggregates which characterize expansion in sense of the outer shape of the root system [55]. Maximum width and minor ellipse axis estimates are onedimensional metrics; convex hull is a function of all three 


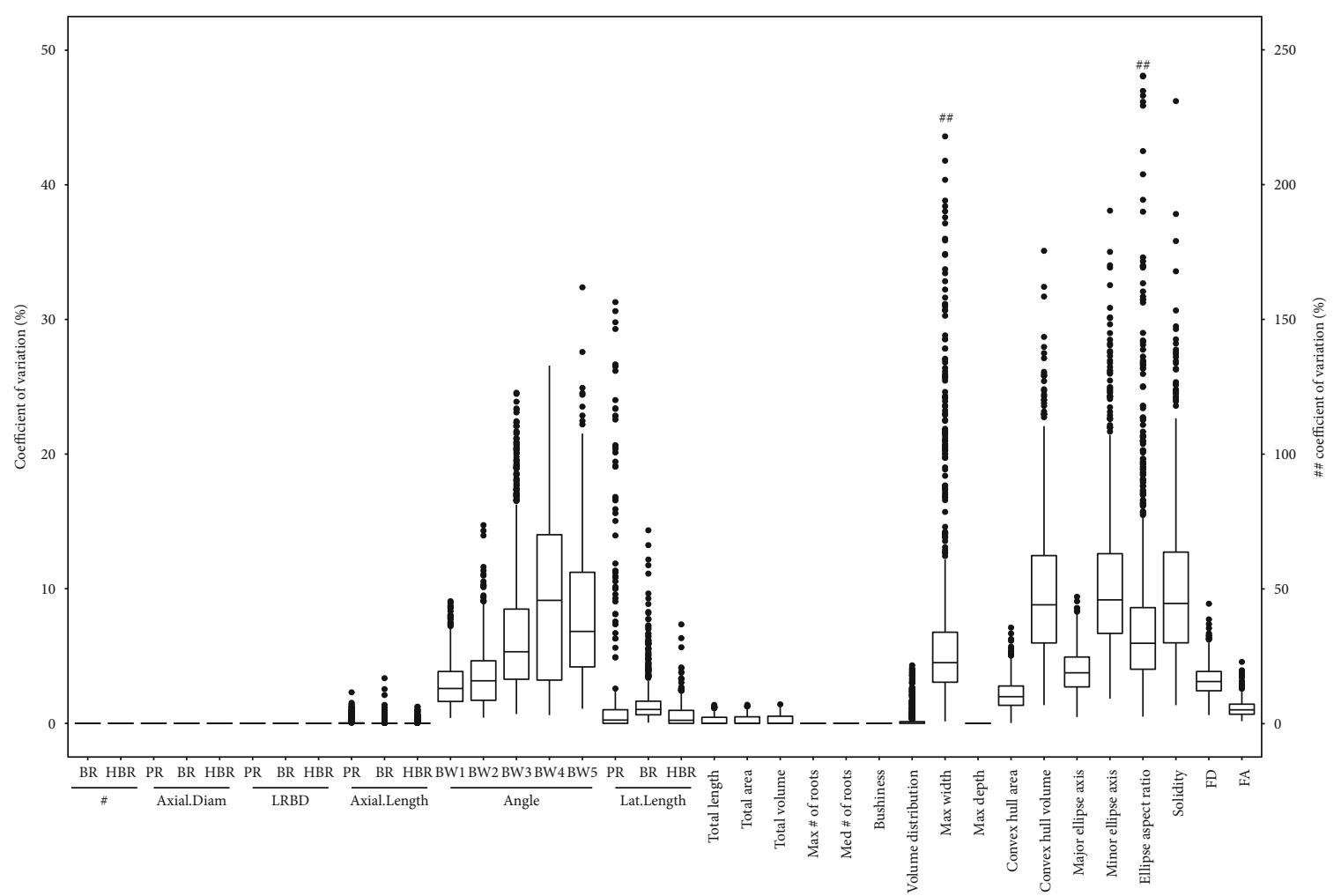

(a)

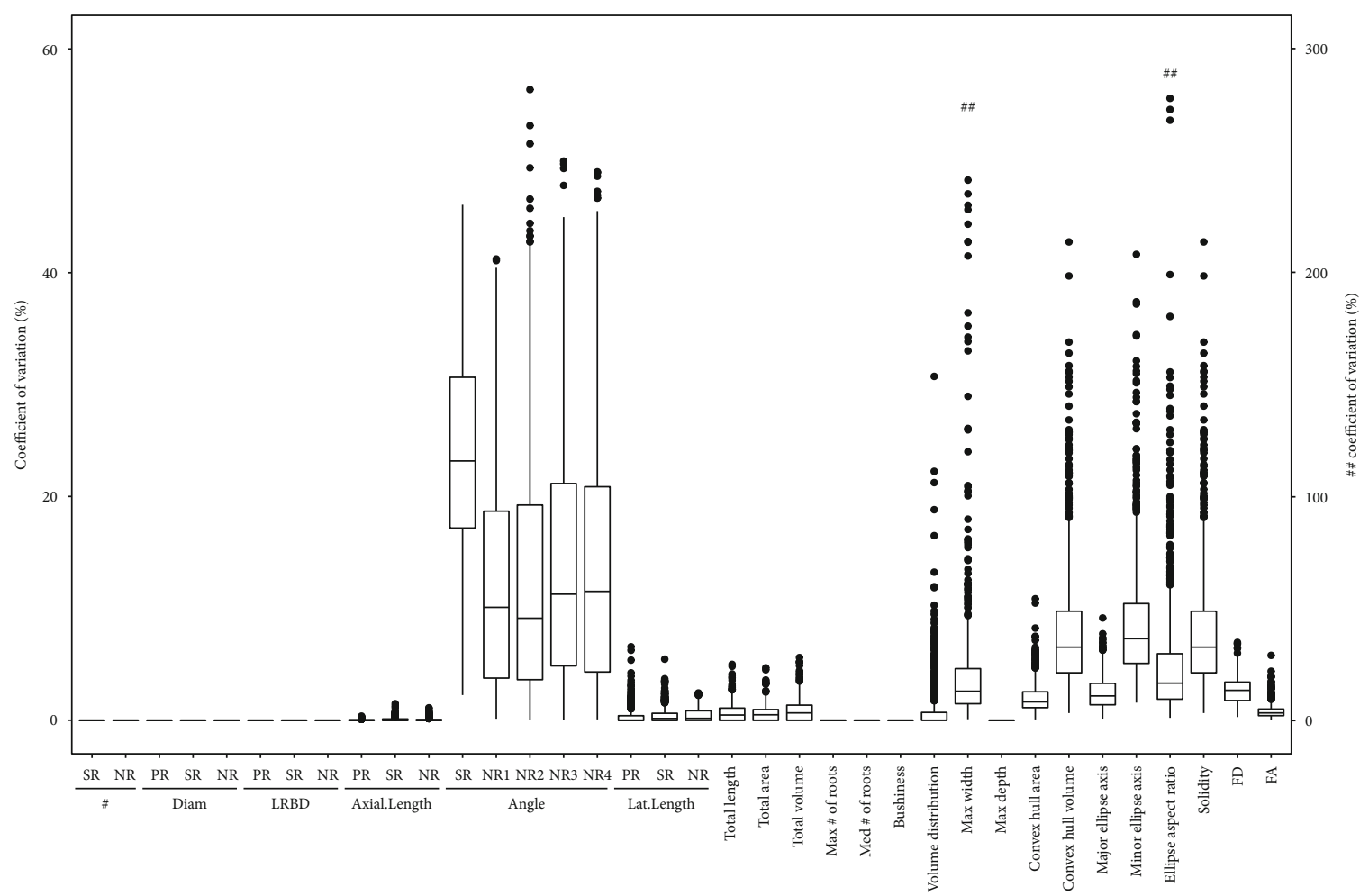

(b)

FIGURE 7: Variation in phene and phene aggregate metrics estimated from rotational series of 2D projected images of 3D bean root system (a) and 3D maize root system (b). BW: basal root; HBR: hypocotyl-borne root; PR: primary root; SR: seminal root; NR: nodal root; \#: number of roots; Axial.Diam: axial root diameter; Axial.Length: axial root length; LRBD: lateral root branching density; Lat.Length: lateral root length; FD: fractal dimension; FA: fractal abundance. 

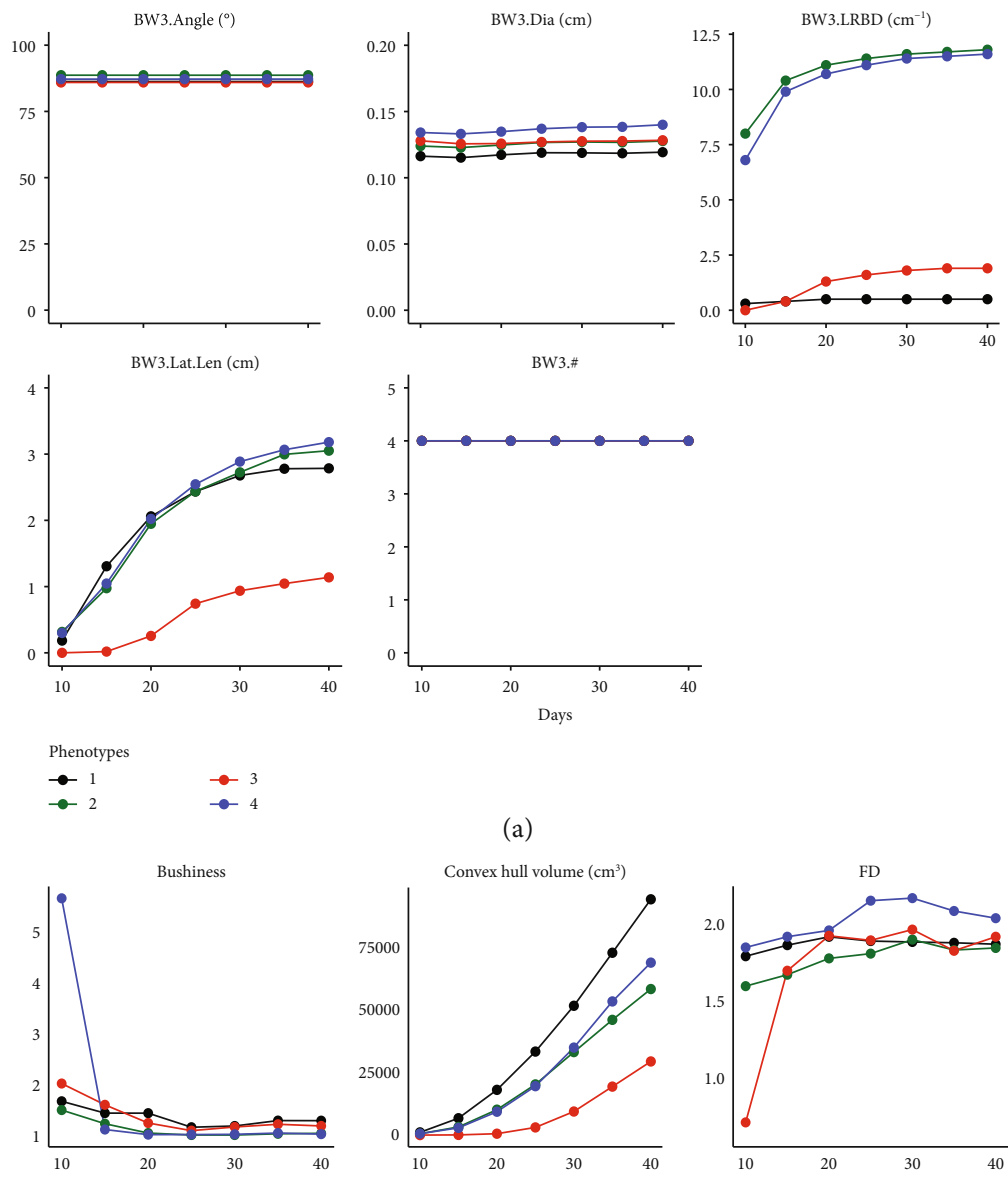

(a)
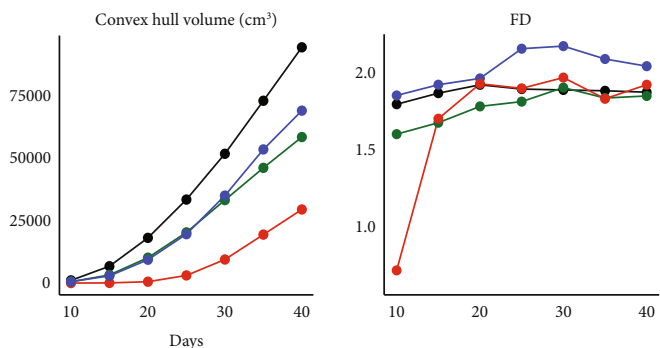

(b)
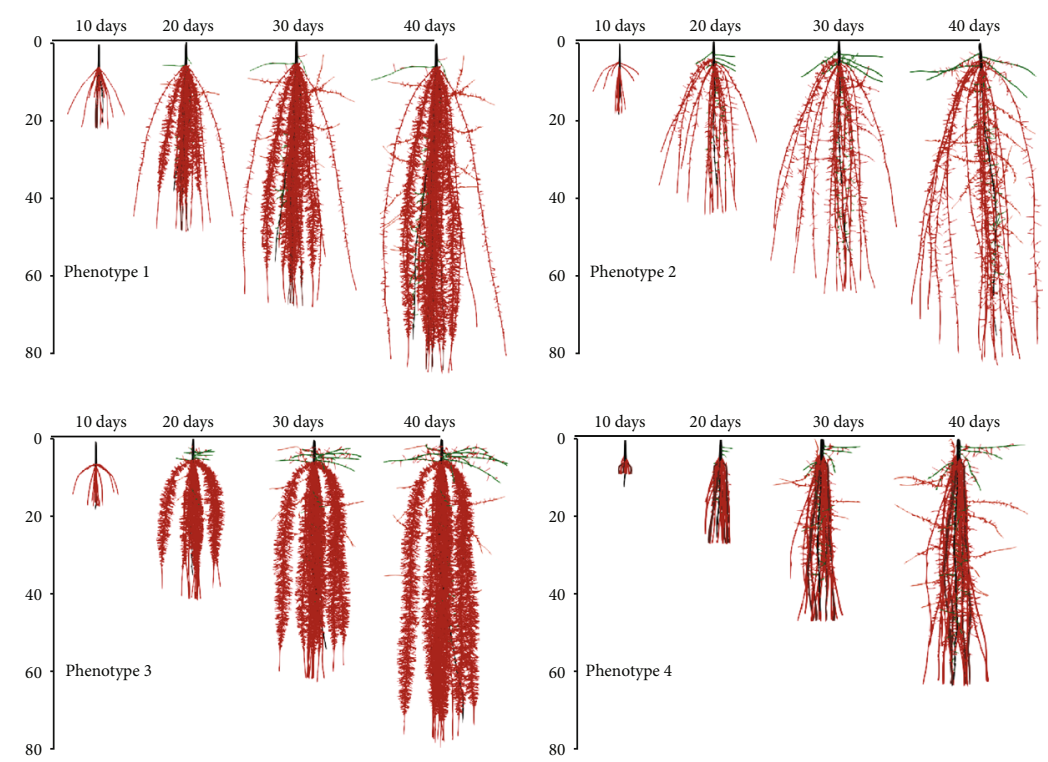

(c)

Figure 8: Trait dynamics of bean root phenotypes over 30 days of growth from day 10 to day 40 . Changes in estimates of phenes associated with basal whorl 3 (BW3) are shown in (a). Similar trends were seen in other root classes (Supplementary Figure S4(a)). Changes in estimates of the phene aggregates bushiness, convex hull volume, and fractal dimension (FD) are shown in (b). Trends in estimates of other phene aggregates included in this study are shown in Supplementary Figure S4(b). The phenotypes for which the metrics are presented in (a, b) are visualized in (c). Primary roots are in black; basal root is in red; hypocotyl-borne roots are in green. BW3: basal roots at whorl 3; Dia: axial root diameter; LRBD: lateral root branching density; Lat.Len: lateral root length; \#: number of axial roots. 

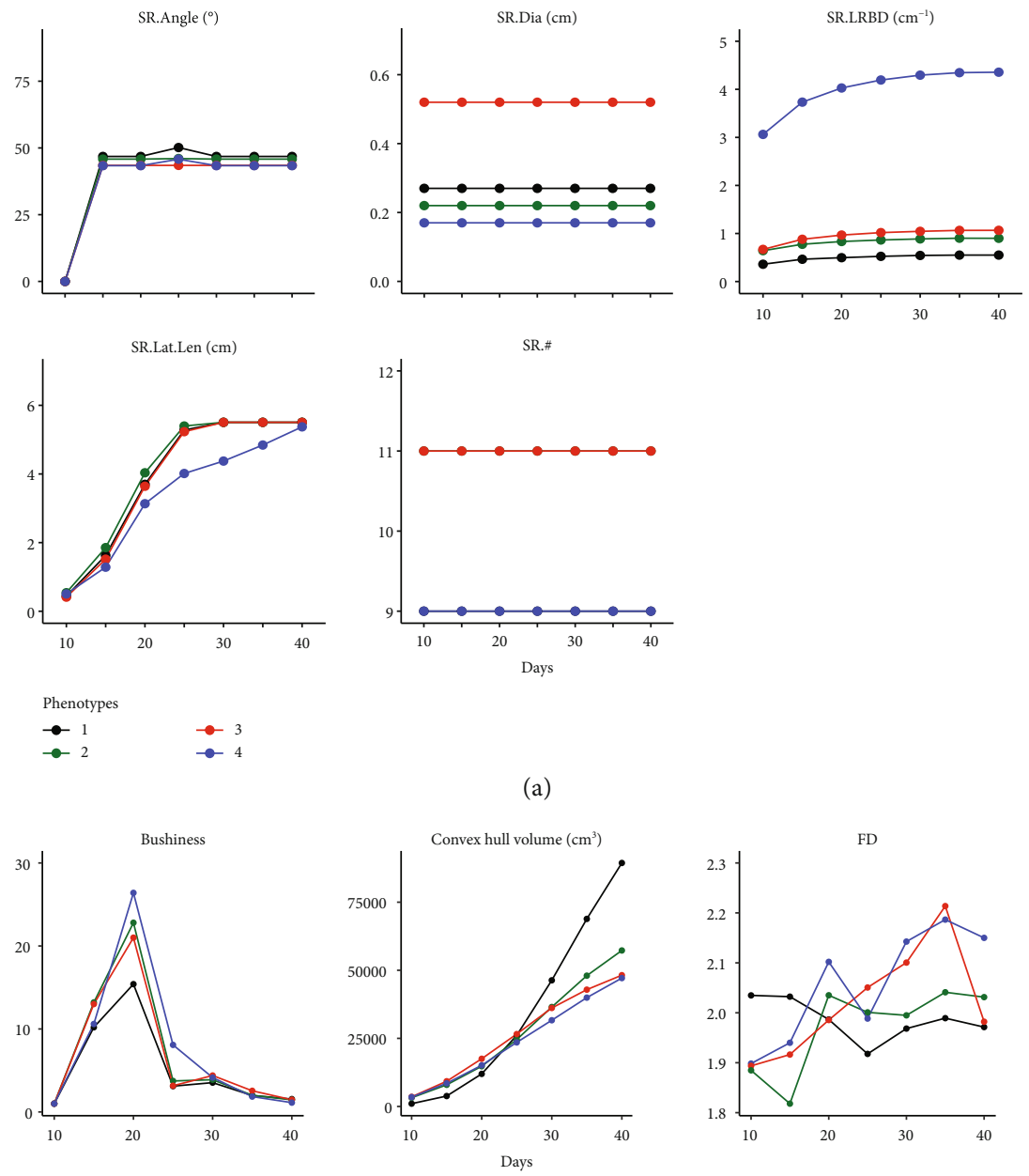

(b)
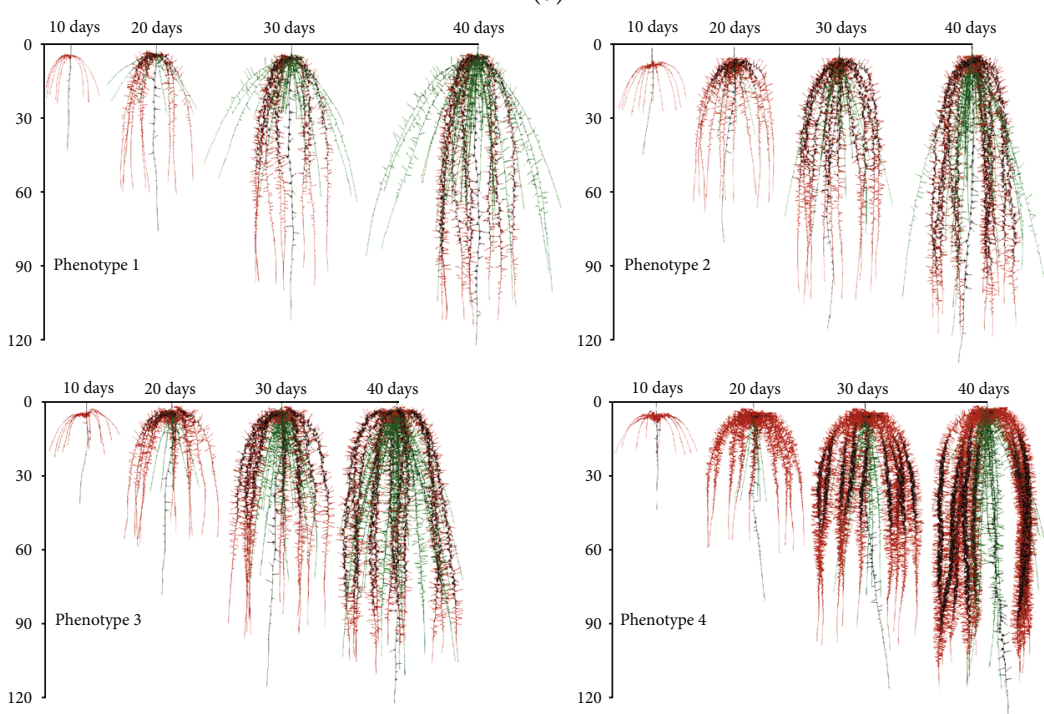

(c)

FIGURE 9: Trait dynamics of maize root phenotypes over 30 days of growth from day 10 to day 40. Changes in estimates of phenes associated with seminal roots (SR) are shown in (a). Similar trends were seen in other root classes (Supplementary Figure S5(a)). Changes in estimates of the phene aggregates bushiness, convex hull volume, and fractal dimension (FD) are shown in (b). Trends in estimates of other phene aggregates included in this study are shown in Supplementary Figure S5(b). The phenotypes for which the metrics are presented in (a, b) are visualized in (c). Primary roots are in black; seminal roots are in red; nodal roots are in green. SR: seminal roots; Dia: axial root diameter; LRBD: lateral root branching density; Lat.Len: lateral root length; \#: number of axial roots. 
dimensions [22]. These differences mean that as the root grows, estimates of the convex hull have a much greater increase in magnitude than does maximum width. Solidity, which is a ratio of the total volume and convex hull, could increase or decrease as total volume is dependent on number of roots and lengths of the roots of different root classes and diameters; however, convex hull estimates the volumetric expansion of the outer shape of the root system.

\subsection{Phene Aggregate Metrics Are Not a Unique Estimate of} Phenotype. Phene aggregate measures such as rooting depth are functionally useful traits, as has been demonstrated by several studies. Rooting depth however is influenced by several phenes including root angle, number of roots, and LRBD (e.g., [25, 27-29, 31]). A measure of rooting depth however does not provide any information on the constituent phenes such as rooting angle and number of roots which all contribute to rooting depth. The same is true for other phene aggregate measures such as convex hull volume. Convex hull, defined as the shape of an object created by joining its outermost points, has been used as an indicator of the extent of soil exploration. Calculating convex hull from point clouds requires minimal preprocessing, making it a popularly used phenotyping metric. Although convex hull can provide interesting information about the overall root system shape [21,56], it was not found to be useful in discriminating between phenotypes of different populations [16]. In a study comparing roots in compacted and uncompacted soil where root geometry is severely affected by soil characteristics, convex hull volume differed by a factor of 3 [43]. Here, we demonstrate that phenotypes with convex hull estimates within as low as $5 \%$ of each other can have phenes expressed in distinctly different states.

While the estimate of a single phene aggregate metric might not be useful in discriminating between phenotypes, using multiple phene aggregate metrics can probably be useful. Each phene aggregate trait gives an estimate of the phenotype by capturing different combinations of phenes. Total length, area, and volume give an estimate of the size of the root system by indirectly measuring the number of roots, length of roots, and the diameter of the roots. Convex hull, minor ellipse axis, major ellipse axis, ellipse aspect ratio, maximum width, and maximum depth provide information of the extent of the shape by providing a measurement root angle and root length. Estimates of these phene aggregates, even though they distinguish features of the root system and complement one another in important ways [17], do not provide any information on the phene states that comprise the phenotype. Studies aimed at finding root traits which discriminate between populations/phenotypes have found that no single phene aggregate trait was important [21]. Which traits were key as well as the number of informative traits were highly dependent on differences between RSA and the imaging day [21]. Complexity of RSA over time reinforces the necessity of assessing a large number of traits to distinguish between different varieties as well as individual varieties at different ages $[16,17,21]$. Accuracy of the differ- ent metrics is strongly linked to the root phenotypes analyzed as well as their size and complexity.

4.4. Variation in Estimates from 2D Projection Images Arises Especially due to Phenes That Determine the Geometry of the Root System. Root angle is an important phene for soil resource capture; studies have shown that shallow root angles are important for capture of immobile soil nutrients and deep root angles for mobile soil nutrients as well as water capture $[7,26,50,57-62]$. Differences in root growth angle result in phenotypes with distinct differences due to trade-offs in the capture of mobile and immobile soil resources and resulting trade-offs in phenes leading to large effects in biomass production $[38,50,63]$. Our results show that estimate of root angle is affected by the $2 \mathrm{D}$ projection of the root system. Root angle determines the geometry of the root system and was found to be an important variable in determining variations in convex hull area, convex hull volume, maximum width, and minor ellipse axis (Table 2). Aggregate phene traits capturing the geometry or overall shape of the root cannot be measured accurately using estimates derived from 2D data. The variation in the estimates of root angle when measured using $2 \mathrm{D}$ projections affects the estimates of all phene aggregate traits in which they play an important role directly or indirectly; these include secondary phene aggregate traits such as solidity, ellipse aspect ratio as well as root complexity traits $F D$ and $F A$ (Figures 8 and 9). Variation is greater in phene aggregates which are estimates of some function of more than one aggregate phene. Even though our root phenotypes are simulated, they are based on empirical parameters, and differences in number of roots, angles of each root class, etc., were varied, and as a result, our root phenotypes were not symmetrical, to replicate actual root system in fields. This is important because most roots found in nature are not symmetrical. We found that greater asymmetry was associated with greater variation in the aggregate phenotypic metrics estimated from $2 \mathrm{D}$ projections. Results from studies using 2D images from gel culture, growth pouches, and narrow growth containers with a transparent face, etc., should be interpreted with caution.

4.5. Variation in Phene Aggregate Metrics with Time Is Species Dependent. We analyzed root phenotypes of two species, maize and common bean, representing a monocot and a dicot root architecture. The main difference between bean, which is a dicot root system, and monocot root systems is that new roots (laterals) emerge from already existing roots in dicots, whereas in monocots nodal roots continually emerge over time from shoot nodes near or above the soil surface [38]. Therefore, the vertical distribution of roots varies between maize and bean, with the bean root system having a relatively equal root distribution whereas maize has more proportion of roots in the topsoil $[64,65]$. The number of roots as well as root diameter depends on the nodal position in maize. This is probably the reason for the great temporal variation in metrics such as volume distribution and bushiness which are related to root size. It has been suggested that metrics accurate for small dicot root systems might fail for large dicot or small monocot root systems [39]. Our study 
confirms that estimates of phene aggregates are not only dependent on phenotype and time but also on the plant species.

4.6. Metrics of Root Complexity. Fractal parameters are different from all the estimated phene aggregates in that they do not provide information on shape of the phenotype and extent of shape or size of the root system but instead measure the geometric complexity of the root phenotype $[18,19,66]$. All the phenes tested were important in determining fractal estimates. Fractal dimension was useful in differentiating between P inefficient and P efficient bean genotypes [19] as well study of roots fractal parameters with uptake of diffusion limited nutrients and between genotypic variation in wheat and study developmental responses in rice [27, 67]. It was found, however, that not a single but combinations of multiple fractal measurements provide useful information $[19,20]$. Phenotypes with comparable aggregate phene trait estimates can be a result of different combinations of phenes in distinctly different phene states. This implies that estimates of phene aggregate traits measure the aggregate of multiple phenes [12]. Studies have shown that complex phenotypic traits such as root complexity as measured by fractal analysis are determined by a multitude of genes with small effects [68]. Even though several studies have resulted in identification of QTLs for aggregate phene traits [17, 21, 69, 70], only two genes directly controlling RSA have been cloned [26, 54]. Estimates of QTL locations or effects per se do not give us direct biological information regarding the product or function of each gene and the interactions among genes [71]. Phenes are unique, meaning, are the product of only one set of genes and processes at a specified scale of resolution $[7,10]$, and so, phene selection is more genetically tractable than selection for traits that aggregate multiple phenes, because axiomatically phenes are under simpler genetic control than any combination of phenes [7].

4.7. Selection of Phenotypes Based on Phenes Is Useful for Breeding. Several phenes have been studied, and their functional utility has been established including number of roots (crown roots in maize, basal roots in bean), root growth angle (shallow for phosphorus uptake and deep rooting angle for nitrogen capture), lateral root branching density, and length for nitrate uptake $[25,29,38,58,59,72-74]$. In the bean root system, basal roots emerge at the seedling stage, and seedling root phenotypes have significant relationships with mature root phenotypes in the bean root system. Number of basal roots as well as basal root growth angle is stable over time as proven by the fact that studies selecting for basal root number and angle at different stages of growth from seedling to few weeks old plants [75-77] have been consistent. Genetic factors explained $52 \%$ to $57 \%$ of genetic variation of phenes in bean including basal root whorl number, basal root number, adventitious root number, and $52 \%$ of phenotypic variation in taproot length in seedlings [78]. Crown root and brace root number, angle, and LRBD were found to be genotypespecific and did not change across growth stages in maize [25]. Basal diameter remains constant in maize while apical diameter varies; in dicots like bean, diameter increases with age due to secondary root growth [79]. Root growth/elongation rates determine the length of the root and are thought to be phenes $[12,78]$. However, carbon limitations could result in delay of emergence of axial roots as well as play a role in determining the final number of axial roots. DemotesMainard and Pellerin [80] have observed in maize that the emergence of axial roots was delayed, and the final number of axial roots was reduced, with increasing levels of competition for light between plants. Time of emergence of roots could also be an important phene, especially in maize where roots emerge from different nodes over time. Recent studies have shown that cellular anatomy varies among nodes providing evidence for node-specific traits [81]. Our approach using elemental phenes to discriminate between architecturally and anatomically distinct phenotypes based on phene states has been used successfully for selection of functionally superior phenotypes for different crop species [82]. We suggest that it is best to study the phenotypes at their elementary level of organization, namely, phenes in order to get a better understanding of their functional value in terms of the interactions among the phenes and also to identify their genetic features.

\section{Conclusions}

These results demonstrate that phenes including number of roots, diameter of roots, lateral root branching density, and root growth angle provide reliable descriptors of root phenotypes. Phenes are also stable over time and independent of time of phenotyping. Estimates of phenes provide a complete description of the resulting phenotype and also enable easier prediction of functional attributes the phenotype could potentially have. Data from our in silico phenotyping environment provides access to complete information concerning root architectural phenotypes without measurement error, sampling limitations, or confounding factors such as phenotypic plasticity or root loss. Even under these conditions, estimates of aggregate phenotypic metrics are less reliable than those of phene states. Even though the estimates of aggregate phenotypic metrics are dependent on the phenotype, the estimates are not unique estimates of underlying states of the constituent phenes. Estimates of phene aggregates also vary in magnitude at different time points of growth and the magnitude of change being dependent on the aggregate phenotype metric used as well as the constituent phenes. Unlike methods used to estimate aggregate phenotypes, estimation of phenes involves simple, straightforward procedures and yield reliable results. We suggest that measurement of phenes provides data that are more robust, reliable, and relevant than metrics that estimate the aggregation of multiple subtending phene states. We show this in the context of root architectural phenotypes but propose that these concepts apply to phenomic analysis of any organism.

\section{Data Availability}

Data generated in this study is freely available from the corresponding author. The executable code of the version of 
SimRoot employed in this study, parameters used to generate these data, and the raw data are all available at https:// figshare.com/s/58c7599752bcb75fbd76.

\section{Additional Points}

One Sentence Summary. Metrics of phenes are more reliable, stable, and robust descriptions of root architecture than are estimates of phene aggregates.

\section{Conflicts of Interest}

The authors declare that there is no conflict of interest regarding the publication of this article.

\section{Authors' Contributions}

HR conducted the experiments, analyzed the data, and wrote the article. JPL conceived and supervised the project and contributed to data analysis and writing. JPL agrees to serve as the author responsible for contact and ensures communication. HR designed and conducted the research, developed the analytical framework, analyzed the results, and wrote the manuscript. JPL assisted with experimental design, data interpretation, and manuscript preparation.

\section{Acknowledgments}

This research was supported by the National Institute of Food and Agriculture, the U.S. Department of Agriculture Hatch project 4732, and the Foundation for Food and Agriculture Research "Crops in Silico" project.

\section{Supplementary Materials}

Supplementary 1. Supplementary Figure S1: representative images of 2D projections of a mazie roots system rotated by $20^{\circ}, 60^{\circ}, 100^{\circ}, 140^{\circ}, 180^{\circ}, 220^{\circ}, 260^{\circ}, 300^{\circ}$, and $340^{\circ}$. Supplementary Figure S2: cluster heat map of phenotypic traits. Hierarchical clustering of all bean phenotypes was generated using Spearman correlation coefficient of min-max scaled phene values at 40 days (a). The color scale indicates the magnitude of the trait values (blue, low value; red, high value). The numbers indicated on the heat map refer to a representative phenotype in the specific region of the heat map. The corresponding phenotypes are visualized in (b). \#: number of axial roots; Axial.Diam: axial root diameter; LRBD: lateral root branching density; Axial.Length: axial root length; Lat.Length: lateral root length; Lat.Diam: lateral root diameter; BW1: basal roots at whorl 1; BW2: basal roots at whorl 2; BW3: basal roots at whorl 3; BW4: basal roots; BW5: basal roots at whorl 5; HBR: hypocotyl-borne roots; PR: primary roots. Supplementary Figure S3: cluster heat map of phenotypic traits. Hierarchical clustering of all maize phenotypes was generated using Spearman correlation coefficient of min-max scaled phene values of at 40 days (a). The color scale indicates the magnitude of the trait values (blue, low value; red, high value). The numbers indicated on the heat map refer to a representative phenotype in the specific region of the heat map. The corresponding phenotypes are visual- ized in (b). \#: number of axial roots; Axial.Diam: axial root diameter; LRBD: lateral root branching density; Axial.Length: axial root length; Lat.Length: lateral root length; Lat.Diam: lateral root diameter; NR1: nodal roots at position 1; NR2: nodal roots at position 2; NR3: nodal roots at position 3; NR4: nodal roots at position 4; SR: seminal roots; PR: primary roots. Supplementary Figure S4: trait dynamics of bean root phenotypes over 30 days of growth from day 10 to 40 . Change in estimates of phenes (a). Change in estimates of phene aggregates (b). BW1: basal roots at whorl 1; BW2: basal roots at whorl 2; BW4: basal roots at whorl 4; BW5: basal roots at whorl 5; HBR: hypocotyl-borne roots; PR: primary root; Dia: axial root diameter; LRBD: lateral root branching density; Lat.Len: lateral root length; \#: number of axial roots; FA: fractal abundance. Supplementary Figure S5: trait dynamics of maize root phenotypes over 30 days of growth from day 10 to 40 . Change in estimates of phenes (a). Change in estimates of phene aggregates (b). NR1: nodal roots at position 1; NR2: nodal roots at position 2; NR3: nodal roots at position 3; NR4: nodal roots at position 4; PR: primary root; Dia: axial root diameter; LRBD: lateral root branching density; Lat.Len: lateral root length; \#: number of axial roots; FA: fractal abundance.

Supplementary 2. Supplementary Table S1: range of input values for generating bean root phenotypes. PR: primary root; HBR: hypocotyl-borne-root; BW: basal whorl; BW1, BW2, BW3, BW4, and BW5 refer to the position of the basal whorl counted from basipetal to acropetal position; Dia: axial root diameter; Lat.Dia: lateral root diameter; LRBD: lateral root branching density. Supplementary Table S2: range of input values for generating maize root phenotypes. PR: primary root; SR: seminal root; NR: nodal root; NR1, NR2, NR3, and NR4 refer to the nodal root position; Dia: axial root diameter; Lat.Dia: lateral root diameter; LRBD: lateral root branching density. ${ }^{*} \mathrm{NR}$ at different positions was considered to have similar parameters.

\section{References}

[1] FAO, The Future of Food and Agriculture: Trends and Challenges, FAO, Rome, 2017.

[2] D. K. Ray, N. D. Mueller, P. C. West, and J. A. Foley, "Yield trends are insufficient to double global crop production by 2050," PLoS One, vol. 8, no. 6, article e66428, 2013.

[3] D. Tilman, C. Balzer, J. Hill, and B. L. Befort, "Global food demand and the sustainable intensification of agriculture," Proceedings of the National Academy of Sciences of the United States of America, vol. 108, no. 50, pp. 20260 20264, 2011.

[4] T. A. Wise, "Can we feed the world in 2050? A scoping paper to assess the evidence. GDAE working paper Np.13-04," Global Development and Environment Institute, Tufts University, Medford, MA, USA, 2013.

[5] G. Den Herder, G. Van Isterdael, T. Beeckman, and I. De Smet, "The roots of a new green revolution," Trends in Plant Science, vol. 15, no. 11, pp. 600-607, 2010.

[6] J. P. Lynch, "Roots of the second green revolution," Australian Journal of Botany, vol. 55, no. 5, p. 493, 2007. 
[7] J. P. Lynch, "Root phenotypes for improved nutrient capture: an underexploited opportunity for global agriculture," The New Phytologist, vol. 223, no. 2, pp. 548-564, 2019.

[8] A. Q. Villordon, I. Ginzberg, and N. Firon, "Root architecture and root and tuber crop productivity," Trends in Plant Science, vol. 19, no. 7, pp. 419-425, 2014.

[9] J. N. Cobb, G. DeClerck, A. Greenberg, R. Clark, and S. McCouch, "Next-generation phenotyping: requirements and strategies for enhancing our understanding of genotypephenotype relationships and its relevance to crop improvement," Theoretical and Applied Genetics, vol. 126, no. 4, pp. 867-887, 2013.

[10] J. P. Lynch and K. M. Brown, "New roots for agriculture: exploiting the root phenome," Philosophical Transactions of the Royal Society B: Biological Sciences, vol. 367, no. 1595, pp. 1598-1604, 2012.

[11] F. Tardieu, L. Cabrera-Bosquet, T. Pridmore, and M. Bennett, "Plant phenomics, from sensors to knowledge," Current Biology, vol. 27, no. 15, pp. R770-R783, 2017.

[12] L. M. York, E. A. Nord, and J. P. Lynch, "Integration of root phenes for soil resource acquisition," Frontiers in Plant Science, vol. 4, p. 355, 2013.

[13] J. A. Atkinson, M. P. Pound, M. J. Bennett, and D. M. Wells, "Uncovering the hidden half of plants using new advances in root phenotyping," Current Opinion in Biotechnology, vol. 55, pp. 1-8, 2019.

[14] R. T. Furbank and M. Tester, "Phenomics - technologies to relieve the phenotyping bottleneck," Trends in Plant Science, vol. 16, no. 12, pp. 635-644, 2011.

[15] R. T. Clark, R. B. MacCurdy, J. K. Jung et al., “Three-dimensional root phenotyping with a novel imaging and software platform," Plant Physiology, vol. 156, no. 2, pp. 455-465, 2011.

[16] A. S. Iyer-Pascuzzi, O. Symonova, Y. Mileyko et al., "Imaging and analysis platform for automatic phenotyping and trait ranking of plant root systems," Plant Physiology, vol. 152, no. 3, pp. 1148-1157, 2010.

[17] C. N. Topp, A. S. Iyer-Pascuzzi, J. T. Anderson et al., “3D phenotyping and quantitative trait locus mapping identify core regions of the rice genome controlling root architecture," Proceedings of the National Academy of Sciences of the United States of America, vol. 110, no. 18, pp. E1695-E1704, 2013.

[18] A. H. Fitter and T. R. Stickland, "Fractal characterization of root system architecture," Functional Ecology, vol. 6, no. 6, p. 632, 1992.

[19] K. L. Nielsen, C. R. Miller, D. Beck, and J. P. Lynch, "Fractal geometry of root systems: field observations of contrasting genotypes of common bean (Phaseolus vulgaris L.) grown under different phosphorus regimes," Plant and Soil, vol. 206, pp. 181-190, 1998.

[20] T. C. Walk, E. Van Erp, and J. P. Lynch, "Modelling applicability of fractal analysis to efficiency of soil exploration by roots," Annals of Botany, vol. 94, no. 1, pp. 119-128, 2004.

[21] P. R. Zurek, C. N. Topp, and P. N. Benfey, "Quantitative trait locus mapping reveals regions of the maize genome controlling root system architecture," Plant Physiology, vol. 167, no. 4, pp. 1487-1496, 2015.

[22] S. Mairhofer, S. Zappala, S. Tracy et al., "Recovering complete plant root system architectures from soil via X-ray $\mu$-computed tomography," Plant Methods, vol. 9, no. 1, p. 8, 2013.

[23] B. Eghball, J. R. Settimi, J. W. Maranville, and A. M. Parkhurst, "Fractal analysis for morphological description of corn roots under nitrogen stress," Agronomy Journal, vol. 85, no. 2, pp. 287-289, 1993.

[24] A. M. Manschadi, J. Christopher, P. DeVoil, and G. L. Hammer, "The role of root architectural traits in adaptation of wheat to water-limited environments," Functional Plant Biology, vol. 33, no. 9, pp. 823-837, 2006.

[25] S. Trachsel, S. M. Kaeppler, K. M. Brown, and J. P. Lynch, "Maize root growth angles become steeper under low N conditions," Field Crops Research, vol. 140, pp. 18-31, 2013.

[26] Y. Uga, K. Okuno, and M. Yano, "Dro1, a major QTL involved in deep rooting of rice under upland field conditions," Journal of Experimental Botany, vol. 62, no. 8, pp. 2485-2494, 2011.

[27] A. M. Manschadi, G. L. Hammer, J. T. Christopher, and P. DeVoil, "Genotypic variation in seedling root architectural traits and implications for drought adaptation in wheat (Triticum aestivum L.)," Plant and Soil, vol. 303, no. 1-2, pp. 115129, 2008.

[28] Y. Gao and J. P. Lynch, "Reduced crown root number improves water acquisition under water deficit stress in maize (Zea mays L.)," Journal of Experimental Botany, vol. 67, no. 15, pp. 4545-4557, 2016.

[29] P. Saengwilai, X. Tian, and J. P. Lynch, "Low crown root number enhances nitrogen acquisition from low nitrogen soils in maize (Zea mays L.)," Plant Physiology, vol. 166, pp. 1-34, 2014.

[30] J. A. Postma, A. Dathe, and J. P. Lynch, “The optimal lateral root branching density for maize depends on nitrogen and phosphorus availability," Plant Physiology, vol. 166, no. 2, pp. 590-602, 2014.

[31] A. Zhan, H. Schneider, and J. P. Lynch, "Reduced lateral root branching density improves drought tolerance in maize," Plant Physiology, vol. 168, no. 4, pp. 1603-1615, 2015.

[32] J. P. Lynch, "Root phenes for enhanced soil exploration and phosphorus acquisition: tools for future crops," Plant Physiology, vol. 156, no. 3, pp. 1041-1049, 2011.

[33] J. G. Chimungu, K. M. Brown, and J. P. Lynch, "Large root cortical cell size improves drought tolerance in maize," Plant Physiology, vol. 166, no. 4, pp. 2166-2178, 2014.

[34] R. E. Jaramillo, E. A. Nord, J. G. Chimungu, K. M. Brown, and J. P. Lynch, "Root cortical burden influences drought tolerance in maize," Annals of Botany, vol. 112, no. 2, pp. 429-437, 2013.

[35] J. P. Lynch, K. L. Nielsen, R. D. Davis, and A. G. Jablokow, "SimRoot : modelling and visualization of root systems," Plant and Soil, vol. 188, no. 1, pp. 139-151, 1997.

[36] L. Breiman, "Random forests," Machine Learning, vol. 45, no. 1, pp. 5-32, 2001.

[37] D. R. Cutler, T. C. Edwards Jr., K. H. Beard et al., "Random forests for classification in ecology," Ecology, vol. 88, no. 11, pp. 2783-2792, 2007.

[38] H. Rangarajan, J. A. Postma, and J. P. Lynch, "Co-optimization of axial root phenotypes for nitrogen and phosphorus acquisition in common bean," Annals of Botany, vol. 122, no. 3, pp. 485-499, 2018.

[39] G. Lobet, I. T. Koevoets, M. Noll et al., "Using a structural root system model to evaluate and improve the accuracy of root image analysis pipelines," Frontiers in Plant Science, vol. 8, p. 447, 2017.

[40] R. Metzner, A. Eggert, D. van Dusschoten et al., "Direct comparison of MRI and X-ray CT technologies for 3D imaging of root systems in soil: potential and challenges for root trait quantification," Plant Methods, vol. 11, no. 1, p. 17, 2015. 
[41] S. J. Mooney, T. P. Pridmore, J. Helliwell, and M. J. Bennett, "Developing X-ray computed tomography to non-invasively image 3-D root systems architecture in soil," Plant and Soil, vol. 352, no. 1-2, pp. 1-22, 2012.

[42] H. Schulz, J. A. Postma, D. van Dusschoten, H. Scharr, and S. Behnke, "Plant root system analysis from MRI images," Computer Vision, Imaging and Computer Graphics, vol. 359, pp. 411-425, 2013.

[43] S. R. Tracy, C. R. Black, J. A. Roberts et al., "Quantifying the impact of soil compaction on root system architecture in tomato (Solanum lycopersicum) by X-ray micro-computed tomography," Annals of Botany, vol. 110, no. 2, pp. 511-519, 2012.

[44] A. Bucksch, J. Burridge, L. M. York et al., "Image-based highthroughput field phenotyping of crop roots," Plant Physiology, vol. 166, no. 2, pp. 470-486, 2014.

[45] M. Landl, A. Schnepf, J. Vanderborght et al., "Measuring root system traits of wheat in 2D images to parameterize $3 \mathrm{D}$ root architecture models," Plant and Soil, vol. 425, no. 1-2, pp. 457-477, 2018.

[46] H. F. Downie, M. O. Adu, S. Schmidt et al., "Challenges and opportunities for quantifying roots and rhizosphere interactions through imaging and image analysis," Plant, Cell \& Environment, vol. 38, no. 7, pp. 1213-1232, 2015.

[47] J. Burridge, C. N. Jochua, A. Bucksch, and J. P. Lynch, "Legume shovelomics: High-Throughput phenotyping of common bean (Phaseolus vulgaris L.) and cowpea (Vigna unguiculata subsp, unguiculata) root architecture in the field," Field Crops Research, vol. 192, pp. 21-32, 2016.

[48] L. Pagès and S. Pellerin, "Evaluation of parameters describing the root system architecture of field grown maize plants (Zea mays L.)," Plant and Soil, vol. 164, no. 2, pp. 169-176, 1994.

[49] S. Pellerin and L. Pagès, "Evaluation of parameters describing the root system architecture of field grown maize plants (Zea mays L.)," Plant and Soil, vol. 164, no. 2, pp. 155-167, 1994.

[50] A. Dathe, J. A. Postma, M. B. Postma-Blaauw, and J. P. Lynch, "Impact of axial root growth angles on nitrogen acquisition in maize depends on environmental conditions," Ann Bot, vol. 118, no. 3, pp. 401-414, 2016.

[51] J. A. Postma and J. P. Lynch, "Theoretical evidence for the functional benefit of root cortical aerenchyma in soils with low phosphorus availability," Annals of Botany, vol. 107, no. 5, pp. 829-841, 2011.

[52] J. A. Postma and J. P. Lynch, "Root cortical aerenchyma enhances the growth of maize on soils with suboptimal availability of nitrogen, phosphorus, and potassium," Plant Physiology, vol. 156, no. 3, pp. 1190-1201, 2011.

[53] T. C. Walk, R. Jaramillo, and J. P. Lynch, "Architectural tradeoffs between adventitious and basal roots for phosphorus acquisition," Plant and Soil, vol. 279, no. 1-2, pp. 347-366, 2006.

[54] M. J. Wedger, C. N. Topp, and K. M. Olsen, "Convergent evolution of root system architecture in two independently evolved lineages of weedy rice," New Phytologist, vol. 223, no. 2, pp. 1031-1042, 2019.

[55] S. Paulus, H. Schumann, H. Kuhlmann, and J. Léon, “Highprecision laser scanning system for capturing 3D plant architecture and analysing growth of cereal plants," Biosystems Engineering, vol. 121, pp. 1-11, 2014.

[56] P. A. Ingram, J. Zhu, A. Shariff, I. W. Davis, P. N. Benfey, and T. Elich, "High-throughput imaging and analysis of root sys- tem architecture in Brachypodium distachyon under differential nutrient availability," Philosophical Transactions of the Royal Society B: Biological Sciences, vol. 367, no. 1595, pp. 1559-1569, 2012.

[57] A. Dathe, J. A. Postma, and J. P. Lynch, "Modeling resource interactions under multiple edaphic Stresses," American Society of Agronomy, Crop Science Society of America, Soil Science Society of America, vol. 4, pp. 1-30, 2015.

[58] J. P. Lynch, "Steep, cheap and deep: an ideotype to optimize water and $\mathrm{N}$ acquisition by maize root systems," Annals of Botany, vol. 112, no. 2, pp. 347-357, 2013.

[59] M. A. Miguel, J. A. Postma, and J. P. Lynch, "Phene synergism between root hair length and basal root growth angle for phosphorus acquisition," Plant Physiology, vol. 167, no. 4, pp. 1430-1439, 2015.

[60] M. A. Miguel, A. Widrig, R. F. Vieira, K. M. Brown, and J. P. Lynch, "Basal root whorl number: a modulator of phosphorus acquisition in common bean (Phaseolus vulgaris)," Annals of Botany, vol. 112, no. 6, pp. 973-982, 2013.

[61] F. Omori and Y. Mano, "QTL mapping of root angle in $\mathrm{F}_{2}$ populations from maize B73 $\times$ teosinte Zea luxurians," Plant Root, vol. 1, pp. 57-65, 2007.

[62] J. Zhu, S. M. Kaeppler, and J. P. Lynch, "Topsoil foraging and phosphorus acquisition efficiency in maize (Zea mays)," Functional Plant Biology, vol. 32, no. 8, pp. 749-762, 2005.

[63] Z. Ge, G. Rubio, and J. P. Lynch, "The importance of root gravitropism for inter-root competition and phosphorus acquisition efficiency: results from a geometric simulation model," Plant and Soil, vol. 218, no. 1-2, pp. 159-171, 2000.

[64] J. A. Postma and J. P. Lynch, "Complementarity in root architecture for nutrient uptake in ancient maize/bean and maize/bean/squash polycultures," Annals of Botany, vol. 110, no. 2, pp. 521-534, 2012.

[65] C. Zhang, J. A. Postma, L. M. York, and J. P. Lynch, "Root foraging elicits niche complementarity-dependent yield advantage in the ancient "Three sisters" (maize/bean/squash) polyculture," Annals of Botany, vol. 114, no. 8, pp. 17191733, 2014.

[66] K. L. Nielsen, J. P. Lynch, and H. N. Weiss, "Fractal geometry of bean root systems: correlations between spatial and fractal dimension," American Journal of Botany, vol. 84, no. 1, pp. 26-33, 1997.

[67] H. Wang, J. Siopongco, L. J. Wade, and A. Yamauchi, "Fractal analysis on root systems of rice plants in response to drought stress," Environmental and Experimental Botany, vol. 65, no. 2-3, pp. 338-344, 2009.

[68] T. E. Grift, J. Novais, and M. Bohn, "High-throughput phenotyping technology for maize roots," Biosystems Engineering, vol. 110, no. 1, pp. 40-48, 2011.

[69] J. A. Atkinson, L. U. Wingen, M. Griffiths et al., "Phenotyping pipeline reveals major seedling root growth QTL in hexaploid wheat," Journal of Experimental Botany, vol. 66, no. 8, pp. 2283-2292, 2015.

[70] K. Kenobi, J. A. Atkinson, D. M. Wells et al., "Linear discriminant analysis reveals differences in root architecture in wheat seedlings related to nitrogen uptake efficiency," Journal of Experimental Botany, vol. 68, no. 17, pp. 49694981, 2017.

[71] R. Bernardo, "Molecular markers and selection for complex traits in plants: learning from the last 20 years," Crop Science, vol. 48 , no. 5, pp. 1649-1664, 2008. 
[72] B. Sun, Y. Gao, and J. P. Lynch, "Large crown root number improves topsoil foraging and phosphorus acquisition," Plant Physiology, vol. 177, no. 1, pp. 90-104, 2018.

[73] A. Zhan and J. P. Lynch, "Reduced frequency of lateral root branching improves $\mathrm{N}$ capture from low- $\mathrm{N}$ soils in maize," Journal of Experimental Botany, vol. 66, no. 7, pp. 20552065, 2015.

[74] J. Zhu, S. M. Kaeppler, and J. P. Lynch, "Mapping of QTLs for lateral root branching and length in maize (Zea mays L.) under differential phosphorus supply," Theoretical and Applied Genetics, vol. 111, no. 4, pp. 688-695, 2005.

[75] H. Liao, G. Rubio, X. Yan, A. Cao, K. M. Brown, and J. P. Lynch, "Effect of phosphorus availability on basal root shallowness in common bean," Plant and Soil, vol. 232, no. 1/2, pp. 69-79, 2001.

[76] R. F. Vieira, J. E. S. Carneiro, and J. P. Lynch, "Root traits of common bean genotypes used in breeding programs for disease resistance," Pesquisa Agropecuária Brasileira, vol. 43, no. 6, pp. 707-712, 2008.

[77] R. F. Vieira and J. P. Lynch, "Root gravitropism of genotypes of common beans used for breeding in Brazil," vol. 4899, pp. 2-3, 2001.

[78] C. F. Strock, J. Burridge, A. S. F. Massas et al., "Seedling root architecture and its relationship with seed yield across diverse environments in Phaseolus vulgaris," Field Crops Research, vol. 237, pp. 53-64, 2019.

[79] C. F. Strock, L. Morrow de la Riva, and J. P. Lynch, "Reduction in root secondary growth as a strategy for phosphorus acquisition," Plant Physiology, vol. 176, no. 1, pp. 691-703, 2018.

[80] S. Demotes-Mainard and S. Pellerin, "Effect of mutual shading on the emergence of nodal roots and the root/shoot ratio of maize," Plant and Soil, vol. 147, no. 1, pp. 87-93, 1992.

[81] J. T. Yang, H. M. Schneider, K. M. Brown, and J. P. Lynch, "Genotypic variation and nitrogen stress effects on root anatomy in maize are node specific," Journal of Experimental Botany, vol. 70, no. 19, pp. 5311-5325, 2019.

[82] J. D. Burridge, H. M. Schneider, B. L. Huynh, P. A. Roberts, A. Bucksch, and J. P. Lynch, "Genome-wide association mapping and agronomic impact of cowpea root architecture," Theoretical and Applied Genetics, vol. 130, no. 2, pp. 419-431, 2017. 